\title{
The Cope Reaction Families: To be or not to be a Biradical
}

\section{Armando Navarro-Vázquez, ${ }^{a}$ Matthias Prall, ${ }^{\mathrm{a}}$ and Peter R. Schreiner ${ }^{\star a, b}$}

${ }^{a}$ Institute of Organic Chemistry, Justus-Liebig-University, Heinrich-Buff-Ring 58, D-35392 Giessen, Germany and ${ }^{b}$ Department of Chemistry, The University of Georgia, Athens, GA 30602-2556,USA,prs@chem.uga.edu

prsdorg.chemie.uni-giessen.de

Absolute Energies (a.u)at the BLYP and BD(T) levels of theory, zero point BLYP vibrational energies, and $\left\langle\mathrm{S}^{2}\right\rangle$ expectation values (a.u.) for unrestricted calculations (UBLYP and UHF for BD(T))

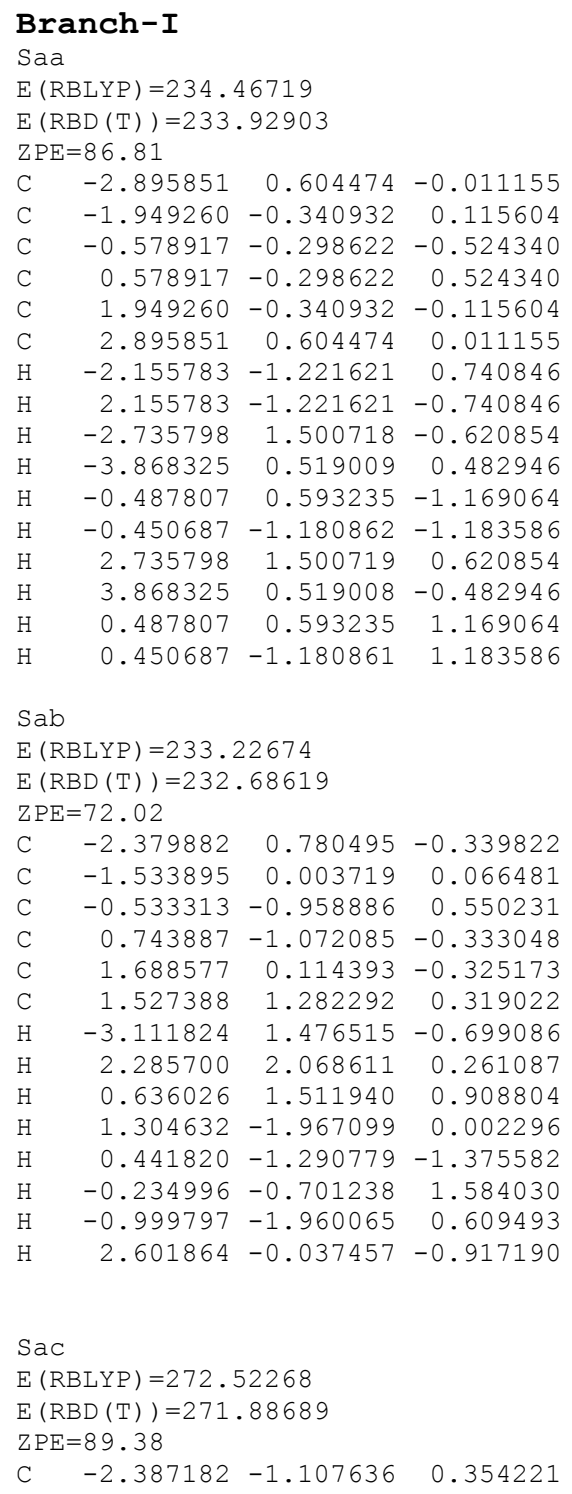

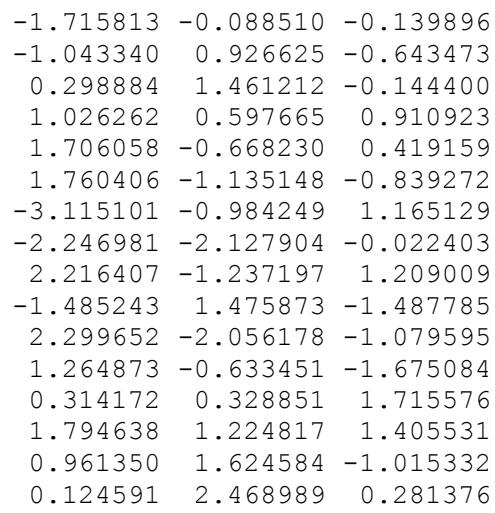




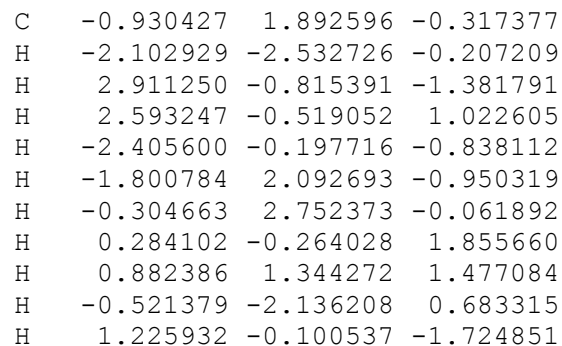

Sae

$E(R B L Y P)=271.30266$

$\mathrm{E}(\operatorname{RBD}(\mathrm{T}))=270.66741$

$\mathrm{ZPE}=74.71$

$\begin{array}{lrrr}\mathrm{C} & -2.148693 & -1.483696 & 0.101031 \\ \mathrm{C} & -1.425935 & -0.499082 & 0.075824 \\ \mathrm{C} & -0.555230 & 0.640831 & 0.100272 \\ \mathrm{C} & 0.718545 & 0.522527 & 0.942065 \\ \mathrm{C} & 1.830249 & -0.332242 & 0.345533 \\ \mathrm{C} & 1.888382 & -0.817393 & -0.904984 \\ \mathrm{H} & -2.789806 & -2.342979 & 0.098189 \\ \mathrm{H} & 2.741260 & -1.413554 & -1.241848 \\ \mathrm{H} & 1.092998 & -0.642809 & -1.634813 \\ \mathrm{H} & 1.113110 & 1.539009 & 1.134788 \\ \mathrm{H} & 0.455143 & 0.110996 & 1.935397 \\ \mathrm{H} & 2.657772 & -0.538149 & 1.037776 \\ \mathrm{C} & -0.858381 & 1.768897 & -0.589335 \\ \mathrm{H} & -0.195199 & 2.638077 & -0.564519 \\ \mathrm{H} & -1.768901 & 1.850355 & -1.187409\end{array}$

Saf

$E(R B L Y P)=310.60242$

$\mathrm{E}(\operatorname{RBD}(\mathrm{T}))=309.87241$

$\mathrm{ZPE}=92.30$

$\begin{array}{lrrr}\mathrm{C} & -2.861337 & -0.673016 & 0.078800 \\ \mathrm{C} & -1.887353 & 0.177735 & -0.156228 \\ \mathrm{C} & -0.910793 & 1.040251 & -0.403785 \\ \mathrm{C} & 0.458225 & 0.991860 & 0.153721 \\ \mathrm{C} & 0.836143 & -0.176041 & 1.058675 \\ \mathrm{C} & 1.226990 & -1.468831 & 0.348068 \\ \mathrm{C} & 1.477726 & -1.625057 & -0.961553 \\ \mathrm{H} & -3.566509 & -0.534692 & 0.907933 \\ \mathrm{H} & -3.013417 & -1.563651 & -0.543674 \\ \mathrm{H} & 1.320644 & -2.336031 & 1.015934 \\ \mathrm{H} & -1.123701 & 1.882096 & -1.077756 \\ \mathrm{H} & 1.768436 & -2.596580 & -1.371579 \\ \mathrm{H} & 1.402529 & -0.794337 & -1.668983 \\ \mathrm{H} & -0.007447 & -0.399196 & 1.739176 \\ \mathrm{H} & 1.674875 & 0.127988 & 1.714335 \\ \mathrm{C} & 1.344493 & 1.972779 & -0.145979 \\ \mathrm{H} & 2.361524 & 1.968130 & 0.255239 \\ \mathrm{H} & 1.078496 & 2.808200 & -0.800942\end{array}$

Sag

$E(R B L Y P)=233.23918$

$\mathrm{E}(\operatorname{RBD}(\mathrm{T}))=232.68955$

$\mathrm{ZPE}=71.89$

$\begin{array}{lrrr}\text { C } & 2.820312 & 0.387333 & -0.332815 \\ \text { C } & 1.642766 & 0.067653 & 0.161256 \\ \text { C } & 0.465527 & -0.263727 & 0.652215 \\ \text { C } & -0.619544 & -1.008117 & -0.115921 \\ \text { C } & -1.931318 & -0.251776 & -0.276385 \\ \text { C } & -2.143933 & 1.044740 & 0.002786\end{array}$

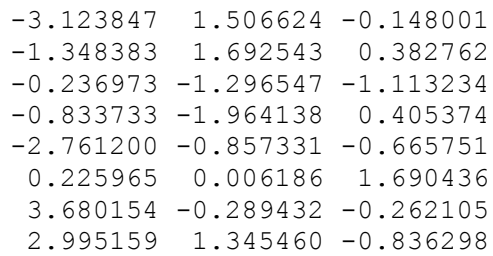

$\mathrm{Sbc}$

$E(R B L Y P)=271.28640$

$E(\operatorname{RBD}(T))=270.64659$

$\mathrm{ZPE}=74.54$

$\begin{array}{lrrr}\text { C } & 2.148683 & 1.363272 & 0.307040 \\ \text { C } & 1.651317 & 0.228347 & -0.137529 \\ \text { C } & 1.156838 & -0.905831 & -0.590834 \\ \text { C } & -0.147444 & -1.560092 & -0.145057 \\ \text { C } & -1.045816 & -0.691246 & 0.781359 \\ \text { C } & -1.678082 & 0.449097 & 0.102844\end{array}$ 


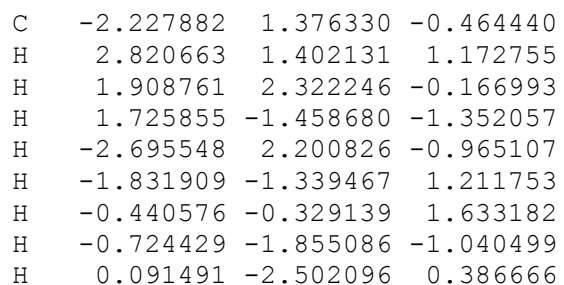

Sbd

$\mathrm{E}(\mathrm{RBLYP})=271.30487$

$E(\operatorname{RBD}(T))=270.67022$

$\mathrm{ZPE}=75.00$

$\begin{array}{lrrr}\text { C } & 0.773375 & 2.012787 & 0.138342 \\ \text { C } & 1.354082 & 0.842403 & -0.207797 \\ \text { C } & 0.828289 & -0.508540 & 0.043759 \\ \text { C } & -0.497781 & -0.671386 & 0.807291 \\ \text { C } & -1.689029 & -0.237395 & 0.056482 \\ \text { C } & -2.683985 & 0.106354 & -0.556034 \\ \text { H } & -3.548583 & 0.414913 & -1.109786 \\ \text { H } & 1.255670 & 2.967456 & -0.087540 \\ \text { H } & -0.199814 & 2.065092 & 0.632770 \\ \text { H } & 2.322424 & 0.868614 & -0.723936 \\ \text { C } & 1.516129 & -1.602606 & -0.362699 \\ \text { H } & 1.151076 & -2.617291 & -0.182083 \\ \text { H } & 2.468236 & -1.512510 & -0.894541 \\ \text { H } & -0.615053 & -1.732195 & 1.092306 \\ \text { H } & -0.440441 & -0.103788 & 1.756742\end{array}$

Sbe

$\mathrm{E}(\mathrm{RBLYP})=270.06380$

$\mathrm{E}(\mathrm{RBD}(\mathrm{T}))=269.42445$

$\mathrm{ZPE}=59.85$

$\begin{array}{lrrr}\text { C } & 1.767304 & 1.849485 & 0.044595 \\ \text { C } & 1.287920 & 0.726270 & 0.027511 \\ \text { C } & 0.686931 & -0.572919 & 0.052362 \\ \text { C } & -0.683865 & -0.689103 & 0.753273 \\ \text { C } & -1.756826 & 0.053519 & 0.073693 \\ \mathrm{C} & -2.647460 & 0.663236 & -0.489559 \\ \text { H } & 2.200250 & 2.830360 & 0.037487 \\ \text { H } & -3.423689 & 1.204930 & -0.993021 \\ \text { H } & -0.952221 & -1.758647 & 0.823830 \\ \text { H } & -0.584332 & -0.320360 & 1.792338 \\ \text { C } & 1.291684 & -1.653704 & -0.496488 \\ \text { H } & 0.819190 & -2.639281 & -0.470137 \\ \text { H } & 2.266674 & -1.577706 & -0.982815\end{array}$

$\mathrm{Sbf}$

$E(R B L Y P)=309.36398$

$\mathrm{E}(\mathrm{RBD}(\mathrm{T}))=308.63020$

$\mathrm{ZPE}=77.34$

$\begin{array}{llll}\text { C } & 2.784164 & 0.437468 & 0.162537\end{array}$

C $\quad 1.774592-0.357953-0.110807$

C $\quad 0.762650-1.166856-0.395908$

$\begin{array}{llll}\text { C } & -0.631515 & -1.003452 & 0.059776\end{array}$

$\begin{array}{llll}\text { C } & -0.984705 & 0.245143 & 0.883615\end{array}$

$\begin{array}{llll}\text { C } & -1.042180 & 1.481606 & 0.084469\end{array}$

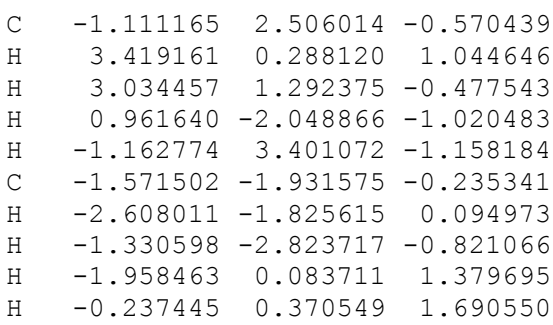

Sbg

$\mathrm{E}(\mathrm{RBLYP})=231.99919$

$E(\operatorname{RBD}(T))=231.44593$

$\mathrm{ZPE}=56.91$

$\begin{array}{rrrr}\text { C } & -1.169801 & -2.125530 & 0.000000 \\ \text { C } & -1.235391 & -0.910159 & 0.000000 \\ \text { C } & -1.336705 & 0.554071 & 0.000000 \\ \text { C } & 0.000000 & 1.309091 & 0.000000 \\ \text { C } & 1.199410 & 0.766479 & 0.000000 \\ \text { C } & 2.405050 & 0.240887 & 0.000000 \\ \text { H } & -1.096120 & -3.195084 & 0.000000 \\ \text { H } & -1.924595 & 0.885913 & 0.879771 \\ \mathrm{H} & -1.924595 & 0.885913 & -0.879771 \\ \mathrm{H} & -0.095031 & 2.403571 & 0.000000 \\ \mathrm{H} & 2.932481 & 0.005325 & 0.931768 \\ \mathrm{H} & 2.932481 & 0.005325 & -0.931768\end{array}$

$\mathrm{Sbh}$

$\mathrm{E}(\mathrm{RBLYP})=270.06552$

$\mathrm{E}(\mathrm{RBD}(\mathrm{T}))=269.41134$

$\mathrm{ZPE}=59.92$

$\begin{array}{lrrr}\text { C } & 1.979164 & 1.783216 & 0.000358 \\ \mathrm{C} & 1.846060 & 0.573548 & -0.000381 \\ \mathrm{C} & 1.711601 & -0.887472 & 0.000000 \\ \mathrm{C} & 0.278452 & -1.424387 & 0.000044 \\ \mathrm{C} & -0.832097 & -0.694560 & 0.000025 \\ \mathrm{C} & -1.910190 & -0.010840 & -0.000002 \\ \mathrm{H} & 2.079102 & 2.850683 & -0.000556 \\ \mathrm{H} & 2.240040 & -1.312223 & 0.878590 \\ \mathrm{H} & 2.240149 & -1.312764 & -0.878263 \\ \mathrm{H} & 0.206531 & -2.520858 & 0.000128 \\ \mathrm{C} & -3.029222 & 0.704583 & -0.000020 \\ \mathrm{H} & -3.011254 & 1.800129 & -0.000050 \\ \mathrm{H} & -4.017176 & 0.230501 & 0.000006\end{array}$

$\mathrm{SCC}$

$E($ RBLYP $)=310.58271$

$\mathrm{E}(\operatorname{RBD}(\mathrm{T}))=309.84749$

$\mathrm{ZPE}=91.92$

C $\quad 2.492018-1.295763-0.621106$

$\begin{array}{llll}\text { C } & 1.880018 & -0.494777 & 0.226399\end{array}$

$\begin{array}{llll}\text { C } & 1.265228 & 0.300701 & 1.077920\end{array}$

$\begin{array}{llll}\text { C } & 0.252874 & 1.391473 & 0.729730\end{array}$

$\begin{array}{llll}\text { C } & -0.252297 & 1.391840 & -0.729120\end{array}$

$\begin{array}{llll}\text { C } & -1.265867 & 0.302313 & -1.077351\end{array}$ 


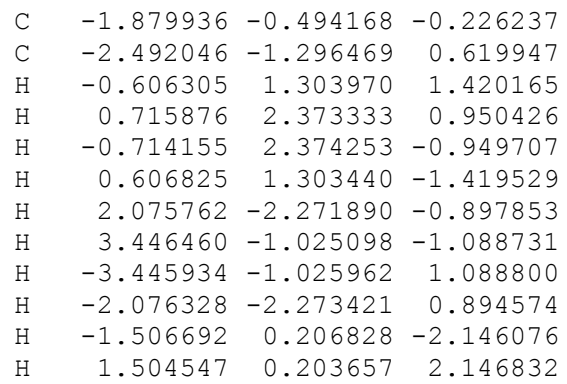

Scd

$\mathrm{E}(\mathrm{RBLYP})=310.60162$

$\mathrm{E}(\mathrm{RBD}(\mathrm{T}))=309.87147$

$\mathrm{ZPE}=92.35$

$\begin{array}{lrrr}\text { C } & -2.557636 & 0.430164 & -1.051179 \\ \mathrm{C} & -1.892754 & 0.039728 & 0.015067 \\ \mathrm{C} & -1.235543 & -0.341032 & 1.091292 \\ \mathrm{C} & 0.231130 & -0.785993 & 1.157039 \\ \mathrm{C} & 1.056811 & -0.552151 & -0.104738 \\ \mathrm{C} & 1.531672 & 0.805353 & -0.426015 \\ \mathrm{C} & 1.309311 & 1.933033 & 0.284881 \\ \mathrm{H} & -3.094111 & -0.280274 & -1.691293 \\ \mathrm{H} & -2.598039 & 1.483612 & -1.352597 \\ \mathrm{H} & 2.136660 & 0.874790 & -1.339430 \\ \mathrm{H} & -1.773474 & -0.376725 & 2.049819 \\ \mathrm{H} & 1.723600 & 2.892323 & -0.036389 \\ \mathrm{H} & 0.703290 & 1.946615 & 1.194441 \\ \mathrm{C} & 1.377116 & -1.588174 & -0.918883 \\ \mathrm{H} & 1.969472 & -1.441271 & -1.827393 \\ \mathrm{H} & 1.054645 & -2.611405 & -0.706684 \\ \mathrm{H} & 0.705174 & -0.270678 & 2.014440 \\ \mathrm{H} & 0.252134 & -1.862552 & 1.410300\end{array}$

Sce

$\mathrm{E}(\mathrm{RBLYP})=309.36133$

$\mathrm{E}(\mathrm{RBD}(\mathrm{T}))=308.62666$

$\mathrm{ZPE}=77.20$

$\begin{array}{lrrr}\mathrm{C} & 2.432022 & 0.970962 & 0.834706 \\ \mathrm{C} & 1.875286 & 0.140659 & -0.020659 \\ \mathrm{C} & 1.326514 & -0.685539 & -0.887675 \\ \mathrm{C} & -0.106715 & -1.224392 & -0.844679 \\ \mathrm{C} & -1.038321 & -0.526668 & 0.148681 \\ \mathrm{C} & -1.442639 & 0.811824 & -0.171190 \\ \mathrm{C} & -1.782643 & 1.939959 & -0.493734 \\ \mathrm{H} & 2.954055 & 0.614838 & 1.730729 \\ \mathrm{H} & 2.390118 & 2.057395 & 0.694188 \\ \mathrm{H} & 1.935345 & -1.050845 & -1.726977 \\ \mathrm{H} & -2.080931 & 2.937197 & -0.750453 \\ \mathrm{H} & -0.533126 & -1.141820 & -1.862458 \\ \mathrm{H} & -0.077472 & -2.305377 & -0.610940 \\ \mathrm{C} & -1.475937 & -1.143537 & 1.273905 \\ \mathrm{H} & -2.144708 & -0.645081 & 1.979131 \\ \mathrm{H} & -1.168677 & -2.165919 & 1.510650\end{array}$

$\operatorname{Scf}$

$E($ RBLYP $)=348.66103$

$E(\operatorname{RBD}(T))=347.83168$

$\mathrm{ZPE}=94.75$

$\begin{array}{llll}\text { C } \quad 2.384028 & -1.397080 & -1.048332\end{array}$

$\begin{array}{llll}\text { C } & 1.795901 & -0.975853 & 0.051052\end{array}$

C $\quad 1.207615-0.568692 \quad 1.156950$

$\begin{array}{llll}\text { C } & 0.280977 & 0.645158 & 1.305727\end{array}$

C $\quad-0.200543 \quad 1.261391-0.002163$

$\begin{array}{lllll}\text { C } & -1.300949 & 0.597414 & -0.732889\end{array}$ $\begin{array}{lrrr}\text { C } & -1.975013 & -0.471014 & -0.329245 \\ \mathrm{C} & -2.648813 & -1.530119 & 0.060958 \\ \mathrm{H} & -0.589637 & 0.330853 & 1.911113 \\ \mathrm{H} & 0.807381 & 1.413914 & 1.901846 \\ \mathrm{H} & 1.911381 & -2.130222 & -1.712836 \\ \mathrm{H} & 3.370599 & -1.029044 & -1.354357 \\ \mathrm{H} & -3.558942 & -1.448450 & 0.668404 \\ \mathrm{H} & -2.331835 & -2.547146 & -0.201872 \\ \mathrm{H} & -1.580190 & 1.052735 & -1.693462 \\ \mathrm{H} & 1.397329 & -1.123332 & 2.087180 \\ \mathrm{C} & 0.355077 & 2.394455 & -0.494748 \\ \mathrm{H} & 0.018842 & 2.836710 & -1.437434 \\ \mathrm{H} & 1.165393 & 2.910015 & 0.027558\end{array}$

$\operatorname{Scg}$

$\mathrm{E}(\mathrm{RBLYP})=271.29426$

$\mathrm{E}(\operatorname{RBD}(\mathrm{T}))=270.64558$

$\mathrm{ZPE}=74.24$

$\begin{array}{lrrr}\mathrm{C} & 1.940103 & 1.499522 & 0.270200 \\ \mathrm{C} & 1.570622 & 0.281232 & -0.063567 \\ \mathrm{C} & 1.239737 & -0.943112 & -0.421297 \\ \mathrm{C} & -0.000054 & -1.729659 & -0.000209 \\ \mathrm{C} & -1.239581 & -0.943056 & 0.421581 \\ \mathrm{C} & -1.570630 & 0.281218 & 0.063750 \\ \mathrm{H} & 1.942083 & -1.497935 & -1.059873 \\ \mathrm{C} & -1.940190 & 1.499397 & -0.270334 \\ \mathrm{H} & 1.661390 & 2.372551 & -0.331315 \\ \mathrm{H} & 2.532486 & 1.696897 & 1.171623 \\ \mathrm{H} & -0.276282 & -2.407661 & -0.832777 \\ \mathrm{H} & 0.275995 & -2.408476 & 0.831739 \\ \mathrm{H} & -1.941540 & -1.497700 & 1.060730 \\ \mathrm{H} & -1.661107 & 2.372674 & 0.330645 \\ \mathrm{H} & -2.533065 & 1.696403 & -1.171516\end{array}$

$\mathrm{Sch}$

$\mathrm{E}(\mathrm{RBLYP})=309.36471$

$E(\operatorname{RBD}(T))=308.61465$

$\mathrm{ZPE}=77.37$

$\begin{array}{lrrr}\text { C } & -2.411433 & -0.393726 & -0.057499 \\ \mathrm{C} & -1.558935 & 0.551960 & -0.157820 \\ \mathrm{C} & -0.683430 & 1.548423 & -0.266349 \\ \mathrm{C} & 0.806491 & 1.472744 & 0.048391 \\ \mathrm{C} & 1.270320 & 0.142630 & 0.625102 \\ \mathrm{C} & 2.262126 & -0.574665 & 0.138206 \\ \mathrm{H} & -1.037873 & 2.532317 & -0.606950 \\ \mathrm{C} & 3.256100 & -1.285187 & -0.351589 \\ \mathrm{H} & 1.383146 & 1.710981 & -0.865203 \\ \mathrm{H} & 1.039649 & 2.289332 & 0.764493 \\ \mathrm{H} & 0.725096 & -0.220699 & 1.506551 \\ \mathrm{H} & 3.093625 & -2.056750 & -1.113392 \\ \mathrm{H} & 4.290499 & -1.139862 & -0.018114 \\ \mathrm{C} & -3.296663 & -1.379084 & 0.046607 \\ \mathrm{H} & -3.006736 & -2.382053 & 0.379525 \\ \mathrm{H} & -4.354866 & -1.231833 & -0.197205\end{array}$

Sdd

$E($ RBLYP $)=310.62165$

$E(\operatorname{RBD}(T))=309.89702$

$\mathrm{ZPE}=92.64$

C $\quad 1.611631-1.385175-1.012762$

C $\quad 1.690623-0.487610-0.006067$

$\begin{array}{llll}\text { C } & 0.664150 & 0.506702 & 0.360706\end{array}$

$\begin{array}{llll}\text { C } & -0.664150 & 0.506697 & -0.360713\end{array}$

$\begin{array}{llll}\text { C } & -1.690614 & -0.487627 & 0.006057\end{array}$

$\begin{array}{llll}\text { C } & -1.611629 & -1.385161 & 1.012780\end{array}$ 


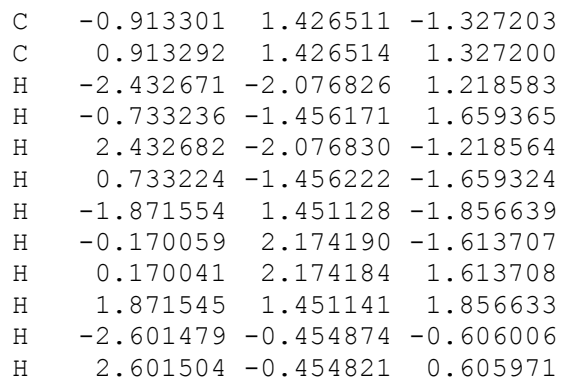

Sde

$E(R B L Y P)=309.38109$

$\mathrm{E}(\mathrm{RBD}(\mathrm{T}))=308.65153$

$\mathrm{ZPE}=77.68$

$\begin{array}{lrrr}\text { C } & 0.953265 & 1.992241 & 0.694380 \\ \mathrm{C} & 1.491783 & 0.966732 & -0.000650 \\ \mathrm{C} & 0.887836 & -0.358661 & -0.231431 \\ \mathrm{C} & -0.528728 & -0.640310 & 0.214064 \\ \mathrm{C} & -1.578897 & 0.195114 & -0.297762 \\ \mathrm{C} & -2.463240 & 0.903147 & -0.754667 \\ \mathrm{H} & -3.243202 & 1.521718 & -1.152867 \\ \mathrm{H} & 1.497020 & 2.932749 & 0.816563 \\ \mathrm{H} & -0.036710 & 1.935045 & 1.152339 \\ \mathrm{H} & 2.492439 & 1.090522 & -0.433964 \\ \mathrm{C} & 1.602295 & -1.342850 & -0.836510 \\ \mathrm{H} & 1.169814 & -2.324627 & -1.040333 \\ \mathrm{H} & 2.638240 & -1.180140 & -1.150115 \\ \mathrm{C} & -0.809619 & -1.676465 & 1.048033 \\ \mathrm{H} & -1.835766 & -1.907502 & 1.343753 \\ \mathrm{H} & -0.009998 & -2.301458 & 1.451882\end{array}$

Sdf

$E(R B L Y P)=348.68000$

$\mathrm{E}(\operatorname{RBD}(\mathrm{T}))=347.85616$

$\mathrm{ZPE}=95.05$

$\begin{array}{lrrr}\text { C } & 2.915852 & 0.350211 & 0.549509 \\ \text { C } & 1.946194 & -0.391035 & 0.064313 \\ \text { C } & 0.979389 & -1.147547 & -0.437332 \\ \text { C } & -0.475352 & -0.957629 & -0.237030 \\ \text { C } & -0.962164 & 0.254544 & 0.518105 \\ \text { C } & -0.883116 & 1.587686 & -0.109053 \\ \text { C } & -0.531721 & 1.853861 & -1.386052 \\ \text { H } & 3.365098 & 0.148316 & 1.529730 \\ \text { H } & 3.313647 & 1.212880 & 0.000836 \\ \text { H } & -1.171268 & 2.420084 & 0.545870 \\ \text { H } & 1.264111 & -2.019199 & -1.043655 \\ \text { H } & -0.523680 & 2.878371 & -1.767271 \\ \text { H } & -0.246301 & 1.065407 & -2.087174 \\ \mathrm{C} & -1.355135 & -1.875787 & -0.710257 \\ \text { H } & -2.432224 & -1.753367 & -0.577846 \\ \text { H } & -1.016489 & -2.768836 & -1.245300 \\ \mathrm{C} & -1.489440 & 0.110642 & 1.760466 \\ \text { H } & -1.858397 & 0.973055 & 2.325143 \\ \text { H } & -1.561535 & -0.866384 & 2.243654\end{array}$

Sdg

$E($ RBLYP $)=271.32123$

$E(\operatorname{RBD}(T))=270.67503$

$\mathrm{ZPE}=75.12$

$\begin{array}{llll}\text { C } & -2.786824 & -0.157312 & 0.000107\end{array}$

$\begin{array}{llll}\text { C } & -1.534666 & -0.560923 & 0.000026\end{array}$

C $\quad-0.307828-1.062385-0.000052$

C $\quad 1.012081-0.384762-0.000048$

C $\quad 1.193175 \quad 1.082293 \quad-0.000005$

$\begin{array}{llll}\text { C } & 0.254654 & 2.052676 & 0.000034\end{array}$

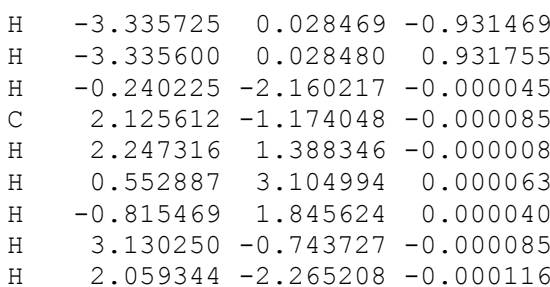

$\mathrm{Sdh}$

$\mathrm{E}(\mathrm{RBLYP})=309.38959$

$\mathrm{E}(\operatorname{RBD}(\mathrm{T}))=308.64215$

$\mathrm{ZPE}=78.14$

$\begin{array}{lrrr}\text { C } & 2.268902 & -0.323823 & 0.000023 \\ \text { C } & 1.035489 & -0.644873 & 0.000029 \\ \text { C } & -0.226338 & -1.097216 & 0.000007 \\ \text { C } & -1.498524 & -0.352796 & -0.000058 \\ \text { C } & -1.606287 & 1.122976 & 0.000047 \\ \text { C } & -0.620165 & 2.044382 & -0.000031 \\ \text { H } & -0.352769 & -2.189701 & 0.000013 \\ \mathrm{C} & -2.654049 & -1.086079 & -0.000025 \\ \text { H } & -2.643803 & 1.480909 & 0.000189 \\ \text { H } & -0.865518 & 3.110226 & 0.000031 \\ \text { H } & 0.437642 & 1.782077 & -0.000158 \\ \text { H } & -3.635632 & -0.605575 & 0.000046 \\ \text { H } & -2.641592 & -2.179016 & 0.000004 \\ \text { C } & 3.549723 & 0.039138 & -0.000007 \\ \text { H } & 4.357021 & -0.701532 & 0.000005 \\ \text { H } & 3.852139 & 1.092360 & -0.000042\end{array}$

See

$E(R B L Y P)=308.14041$

$\mathrm{E}(\operatorname{RBD}(\mathrm{T}))=307.40580$

$\mathrm{ZPE}=62.58$

$\begin{array}{lrrr}\text { C } & 2.091481 & 1.690006 & -0.461145 \\ \text { C } & 1.480296 & 0.672757 & -0.174557 \\ \text { C } & 0.741821 & -0.514259 & 0.146806 \\ \text { C } & -0.741955 & -0.514245 & -0.146818 \\ \text { C } & -1.480514 & 0.672687 & 0.174456 \\ \text { C } & -2.091249 & 1.690169 & 0.461193 \\ \text { H } & 2.633769 & 2.580264 & -0.711979 \\ \text { H } & -2.633665 & 2.580371 & 0.711951 \\ \mathrm{C} & 1.353281 & -1.597157 & 0.696675 \\ \text { H } & 0.780515 & -2.480931 & 0.985464 \\ \text { H } & 2.430142 & -1.607605 & 0.877753 \\ \mathrm{C} & -1.353208 & -1.597179 & -0.696638 \\ \text { H } & -2.430087 & -1.607835 & -0.877678 \\ \text { H } & -0.780380 & -2.480930 & -0.985348\end{array}$

Sef

$E($ RBLYP $)=347.43958$

$\mathrm{E}(\mathrm{RBD}(\mathrm{T}))=346.61080$

$\mathrm{ZPE}=80.05$

$\begin{array}{lrrr}\text { C } & 2.876055 & -0.147174 & 0.591004 \\ \text { C } & 1.802664 & -0.748965 & 0.132508 \\ \text { C } & 0.735084 & -1.379052 & -0.338214 \\ \text { C } & -0.669073 & -0.925270 & -0.241634 \\ \text { C } & -0.972399 & 0.457442 & 0.281778 \\ \text { C } & -0.346683 & 1.574408 & -0.368186\end{array}$ 


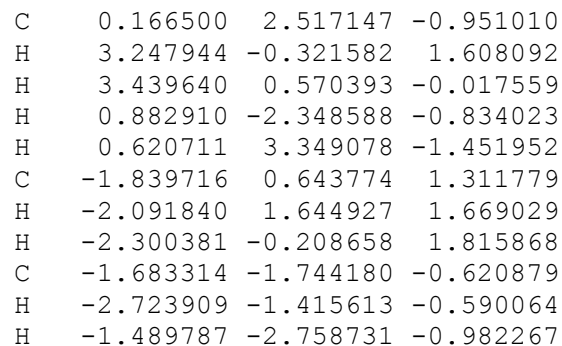

Seg

$\mathrm{E}(\mathrm{RBLYP})=270.08272$

$E(R B D(T))=269.43275$

$\mathrm{ZPE}=59.86$

$\begin{array}{lrrr}\mathrm{C} & -1.083333 & 2.182649 & -0.000047 \\ \mathrm{C} & -1.002368 & 0.965174 & -0.000032 \\ \mathrm{C} & -0.891601 & -0.467361 & -0.000019 \\ \mathrm{C} & 0.463649 & -1.073266 & -0.000251 \\ \mathrm{C} & 1.610320 & -0.409292 & -0.000048 \\ \mathrm{C} & 2.756579 & 0.231075 & 0.000156 \\ \mathrm{H} & -1.161443 & 3.251975 & -0.000046 \\ \mathrm{H} & 0.487737 & -2.171180 & -0.000564 \\ \mathrm{H} & 3.258988 & 0.518092 & -0.931650 \\ \mathrm{H} & 3.259000 & 0.517477 & 0.932145 \\ \mathrm{C} & -2.005402 & -1.253943 & 0.000179 \\ \mathrm{H} & -1.923842 & -2.344133 & 0.000146 \\ \mathrm{H} & -3.007510 & -0.822447 & 0.000329\end{array}$

Seh

$E($ RBLYP $)=308.15091$

$E(\operatorname{RBD}(T))=307.39948$

$\mathrm{ZPE}=62.94$

$\begin{array}{lrrr}\text { C } & -1.247255 & 2.268189 & -0.000124 \\ \mathrm{C} & -1.334260 & 1.051479 & -0.000063 \\ \mathrm{C} & -1.428542 & -0.382828 & -0.000011 \\ \mathrm{C} & -0.185649 & -1.176748 & -0.000313 \\ \mathrm{C} & 1.058624 & -0.681097 & -0.000138 \\ \mathrm{C} & 2.249292 & -0.230942 & 0.000030 \\ \mathrm{H} & -1.174414 & 3.337962 & -0.000168 \\ \mathrm{H} & -0.325604 & -2.266112 & -0.000726 \\ \mathrm{C} & 3.491435 & 0.246769 & 0.000205 \\ \mathrm{H} & 3.692153 & 1.323711 & 0.000619 \\ \mathrm{H} & 4.363806 & -0.415939 & -0.000077 \\ \mathrm{C} & -2.646756 & -1.003434 & 0.000319 \\ \mathrm{H} & -2.720057 & -2.094133 & 0.000351 \\ \mathrm{H} & -3.577226 & -0.433821 & 0.000576\end{array}$

Sff

$E(R B L Y P)=386.73829$

$\mathrm{E}(\operatorname{RBD}(\mathrm{T}))=385.81525$

$\mathrm{ZPE}=97.46$

$\begin{array}{llll}\text { C } & -1.917075 & 2.208839 & 0.754286\end{array}$

C $\quad-0.995195 \quad 1.778662-0.076890$

C $\quad-0.072116 \quad 1.358046-0.930802$

$\begin{array}{lllll}\text { C } & 0.996104 & 0.370799 & -0.655529\end{array}$

$\begin{array}{llll}\text { C } & 0.995968 & -0.371179 & 0.655522\end{array}$

$\begin{array}{llll}\text { C } & -0.072610 & -1.358044 & 0.930788\end{array}$
$\begin{array}{lrrr}\mathrm{C} & -0.995843 & -1.778316 & 0.076875 \\ \mathrm{C} & -1.917889 & -2.208152 & -0.754294 \\ \mathrm{H} & -2.897162 & 1.722646 & 0.835551 \\ \mathrm{H} & -1.746631 & 3.068894 & 1.413698 \\ \mathrm{H} & -1.747802 & -3.068330 & -1.413638 \\ \mathrm{H} & -2.897758 & -1.721533 & -0.835628 \\ \mathrm{H} & -0.062236 & -1.796256 & 1.938780 \\ \mathrm{H} & -0.061580 & 1.796251 & -1.938797 \\ \mathrm{C} & 1.977750 & -0.155837 & 1.566720 \\ \mathrm{H} & 2.003066 & -0.697911 & 2.517339 \\ \mathrm{H} & 2.772505 & 0.571036 & 1.386340 \\ \mathrm{C} & 1.977856 & 0.155143 & -1.566700 \\ \mathrm{H} & 2.772419 & -0.571920 & -1.386264 \\ \mathrm{H} & 2.003476 & 0.697354 & -2.517236\end{array}$

Sfg

$\mathrm{E}(\mathrm{RBLYP})=309.37983$

$E(\operatorname{RBD}(T))=308.63494$

$\mathrm{ZPE}=77.33$

$\begin{array}{lrrr}\text { C } & 1.923744 & -1.905957 & 0.098235 \\ \text { C } & 0.662411 & -1.555710 & -0.015808 \\ \text { C } & -0.631253 & -1.293698 & -0.133904 \\ \mathrm{C} & -1.339536 & -0.000002 & 0.000002 \\ \mathrm{C} & -0.631256 & 1.293695 & 0.133913 \\ \mathrm{C} & 0.662405 & 1.555714 & 0.015799 \\ \mathrm{H} & -1.292026 & -2.148881 & -0.336029 \\ \mathrm{C} & 1.923739 & 1.905960 & -0.098235 \\ \mathrm{H} & 2.605606 & -1.904412 & -0.760860 \\ \mathrm{H} & 2.360511 & -2.187549 & 1.064340 \\ \mathrm{H} & -1.292035 & 2.148881 & 0.336013 \\ \mathrm{H} & 2.605589 & 1.904428 & 0.760869 \\ \mathrm{H} & 2.360517 & 2.187542 & -1.064337 \\ \mathrm{C} & -2.703153 & -0.000004 & -0.000001 \\ \mathrm{H} & -3.275382 & 0.925525 & 0.101202 \\ \mathrm{H} & -3.275379 & -0.925532 & -0.101212\end{array}$

Sfh

$E(R B L Y P)=347.44867$

$E(\operatorname{RBD}(T))=346.60255$

$\mathrm{ZPE}=80.41$

C $\quad-2.069136-0.920727-0.000015$

C $\quad-0.808995-1.103844 \quad 0.000066$

C $\quad 0.498975-1.392463-0.000033$

C $\quad 1.639778-0.463444-0.000033$

C $\quad 1.485320 \quad 1.010137-0.000026$

C $\quad 0.380635 \quad 1.742237-0.000028$

H $\quad 0.782659-2.454520 \quad 0.000255$

C $\quad-0.649187 \quad 2.558800 \quad 0.000039$

$2.437845 \quad 1.559253-0.000201$

$\begin{array}{lll}-1.111318 & 2.906270 & -0.931954\end{array}$

$\begin{array}{lll}-1.110993 & 2.906434 & 0.932138\end{array}$

$-3.376268-0.672504-0.000040$

$\begin{array}{lll}-3.762114 & 0.353591-0.000186\end{array}$

$\begin{array}{lll}-4.119356-1.477377 & 0.000234\end{array}$

$2.902638-0.987799 \quad 0.000007$

$3.788993-0.348725 \quad 0.000336$

$3.071728-2.067288-0.000240$

Sgg

$E(\operatorname{RBLYP})=232.01617$

$E(\operatorname{RBD}(T))=231.45177$

$\mathrm{ZPE}=57.04$

$\begin{array}{llll}\text { C } & 0.741514 & 1.100046 & 0.000049\end{array}$

C $\quad-0.741514 \quad 1.100046-0.000051$

$\mathrm{H} \quad 1.205627 \quad 2.095189 \quad 0.000134$

H $\quad-1.205627 \quad 2.095189-0.000119$

C $\quad 1.538870 \quad 0.040852 \quad 0.000097$

$\begin{array}{llll}\text { C } & -1.538870 & 0.040852 & -0.000098\end{array}$ 


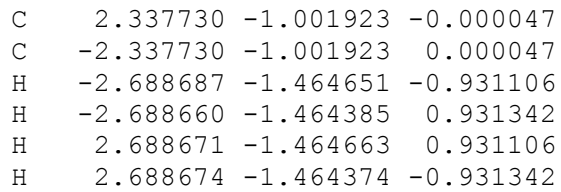

Sgh

$\mathrm{E}(\mathrm{RBLYP})=270.08563$

$\mathrm{E}(\operatorname{RBD}(\mathrm{T}))=269.41934$

$\mathrm{ZPE}=60.17$

$\begin{array}{lrrr}\mathrm{C} & -0.116523 & -1.429799 & 0.000314 \\ \mathrm{C} & -1.521309 & -0.995838 & -0.000329 \\ \mathrm{H} & 0.032485 & -2.517807 & -0.000062 \\ \mathrm{H} & -2.263857 & -1.804801 & -0.000775 \\ \mathrm{C} & 0.967744 & -0.640045 & 0.000198 \\ \mathrm{C} & -1.964906 & 0.258131 & -0.000116 \\ \mathrm{C} & 2.007032 & 0.093854 & 0.000053 \\ \mathrm{C} & -2.412717 & 1.491467 & 0.000128 \\ \mathrm{H} & -2.607833 & 2.037749 & 0.931776 \\ \mathrm{H} & -2.606790 & 2.038489 & -0.931304 \\ \mathrm{C} & 3.093924 & 0.864208 & -0.000086 \\ \mathrm{H} & 3.023782 & 1.957388 & -0.000404 \\ \mathrm{H} & 4.102735 & 0.437119 & -0.000198\end{array}$

Shh

$\mathrm{E}(\mathrm{RBLYP})=308.15575$

$\mathrm{E}(\mathrm{RBD}(\mathrm{T}))=307.38732$

$\mathrm{ZPE}=63.32$

$\begin{array}{lrrr}\mathrm{C} & 0.727555 & 1.635341 & 0.000067 \\ \mathrm{C} & -0.727553 & 1.635339 & 0.000039 \\ \mathrm{H} & 1.197973 & 2.627718 & 0.000301 \\ \mathrm{H} & -1.197968 & 2.627720 & 0.000126 \\ \mathrm{C} & 1.529870 & 0.553392 & -0.000120 \\ \mathrm{C} & -1.529873 & 0.553392 & -0.000141 \\ \mathrm{C} & 2.302876 & -0.454518 & -0.000017 \\ \mathrm{C} & -2.302879 & -0.454517 & -0.000091 \\ \mathrm{C} & 3.112233 & -1.514793 & 0.000036 \\ \mathrm{H} & 2.719342 & -2.537267 & -0.000063 \\ \mathrm{H} & 4.202351 & -1.406984 & 0.000202 \\ \mathrm{C} & -3.112230 & -1.514791 & 0.000071 \\ \mathrm{H} & -2.719339 & -2.537267 & -0.000018 \\ \mathrm{H} & -4.202350 & -1.406993 & 0.000390\end{array}$

Iaa-TS

$E($ RBLYP $)=234.41917$

$E(\operatorname{RBD}(T))=233.87002$

$\mathrm{ZPE}=86.46$

$\begin{array}{llll}\text { C } & 0.000000 & 1.474260 & 0.000000\end{array}$

$\begin{array}{lrrr}\text { C } & 0.000000 & -1.474260 & 0.000000\end{array}$

$\begin{array}{lllll}\text { C } & -0.428327 & 0.917447 & -1.228437\end{array}$

$\begin{array}{llll}\text { C } & -0.428327 & 0.917447 & 1.228437\end{array}$

$\begin{array}{lllll}\text { C } & 0.428327 & -0.917447 & -1.228437\end{array}$

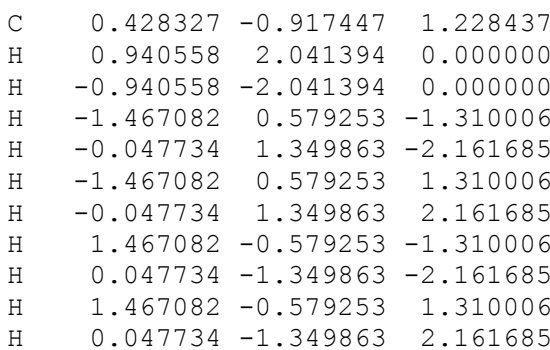

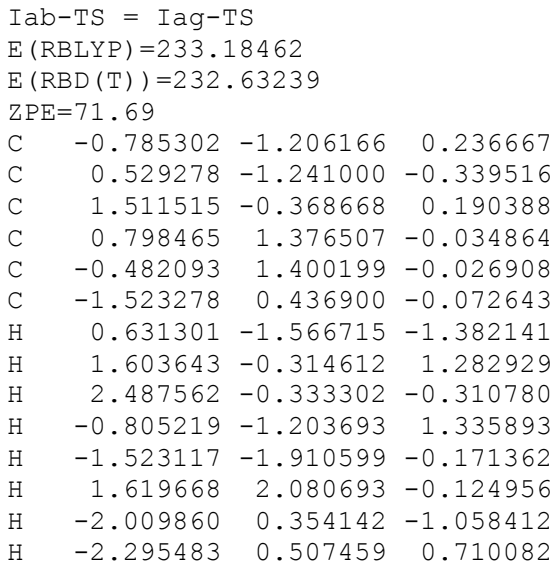

$\mathrm{IaC}-\mathrm{TS}=\mathrm{Iad}-\mathrm{TS}$

$E(R B L Y P)=272.48188$

$E(\operatorname{RBD}(T))=271.84090$

$\mathrm{ZPE}=89.17$

$\begin{array}{llll}\text { C } & 0.253076 & -1.286310 & 0.304526\end{array}$

$-0.977519 \quad 1.263254-0.311647$

$-1.082860-1.287491-0.276173$

$1.148537-0.235070 \quad 0.067964$

$\begin{array}{lll}-1.880401 & 0.219727 & 0.122440\end{array}$

$\begin{array}{lll}0.278952 & 1.383353 & 0.345360\end{array}$

$0.420126-1.896426 \quad 1.202518$

$-1.048427 \quad 1.598443-1.353754$

$-1.115054-1.265928-1.374409$

$\begin{array}{lll}-1.727556 & -2.100208 & 0.092937\end{array}$

$2.445873-0.081454-0.249657$

$\begin{array}{lll}-2.032324 & 0.154381 & 1.209339\end{array}$

$\begin{array}{llll}-2.848943 & 0.181418 & -0.397049\end{array}$

$0.265534 \quad 1.382908 \quad 1.443523$

$0.973316 \quad 2.135095-0.048067$

$\begin{array}{lll}2.961782 & 0.880097-0.175480\end{array}$

$3.037604-0.925831-0.616434$ 


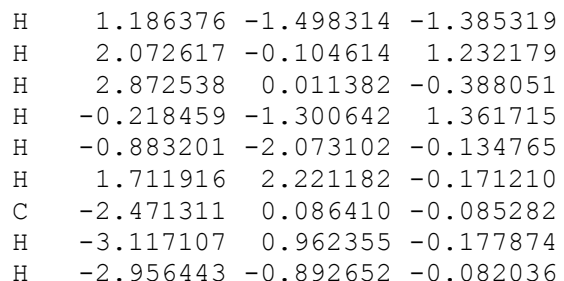

Iaf-TS $(1)=\operatorname{Iaf}-\mathrm{TS}(2)$

$E(R B L Y P)=310.55987$

$E(R B D(T))=309.82286$

$\mathrm{ZPE}=91.93$

$\begin{array}{lrrr}\mathrm{C} & -0.031733 & -1.110250 & 0.201897 \\ \mathrm{C} & 0.060821 & 1.645170 & -0.404214 \\ \mathrm{C} & -1.289333 & -0.402091 & 0.047236 \\ \mathrm{C} & 1.248973 & -0.622662 & 0.070597 \\ \mathrm{C} & -1.248249 & 1.162203 & 0.122469 \\ \mathrm{C} & 1.235130 & 1.308942 & 0.277535 \\ \mathrm{H} & -0.113545 & -2.138179 & 0.577733 \\ \mathrm{H} & 0.118336 & 1.959100 & -1.451646 \\ \mathrm{C} & -2.481131 & -1.033097 & -0.122518 \\ \mathrm{C} & 2.498965 & -1.067501 & -0.191140 \\ \mathrm{H} & -1.379033 & 1.443805 & 1.185134 \\ \mathrm{H} & -2.103260 & 1.576339 & -0.432849 \\ \mathrm{H} & 1.231121 & 1.308633 & 1.374476 \\ \mathrm{H} & 2.190901 & 1.624034 & -0.149897 \\ \mathrm{H} & 3.391161 & -0.568737 & 0.198524 \\ \mathrm{H} & 2.669682 & -1.894046 & -0.889714 \\ \mathrm{H} & -3.421975 & -0.478058 & -0.169113 \\ \mathrm{H} & -2.544046 & -2.117178 & -0.253816\end{array}$

$\mathrm{Ibb}-\mathrm{TS}=\mathrm{Igg}-\mathrm{TS}$

$\mathrm{E}(\mathrm{RBLYP})=231.94818$

$E(R B D(T))=231.39107$

$\mathrm{ZPE}=56.67$

$\begin{array}{lrrr}\text { C } & 0.114348 & -1.406706 & 0.000060 \\ \mathrm{C} & -1.216590 & -0.885217 & -0.000084 \\ \mathrm{C} & -1.218014 & 0.883778 & 0.000015 \\ \mathrm{C} & 0.112303 & 1.406791 & -0.000027 \\ \mathrm{C} & 1.311410 & 1.004785 & 0.000053 \\ \mathrm{C} & 1.312733 & -1.003146 & 0.000042 \\ \mathrm{H} & 2.346186 & 1.318049 & -0.000306 \\ \mathrm{H} & 2.347955 & -1.314972 & -0.000153 \\ \mathrm{H} & -1.798882 & 1.164270 & -0.893917 \\ \mathrm{H} & -1.798551 & 1.163472 & 0.894391 \\ \mathrm{H} & -1.796758 & -1.166310 & -0.894313 \\ \mathrm{H} & -1.797078 & -1.166220 & 0.893946\end{array}$

$\mathrm{Ibc}-\mathrm{TS}=\mathrm{Idg}-\mathrm{TS}$

$E(R B L Y P)=271.24873$

$\mathrm{E}(\operatorname{RBD}(\mathrm{T}))=270.60222$

$\mathrm{ZPE}=74.42$

$\begin{array}{lrrr}\text { C } & 1.067798 & -1.176412 & -0.311002 \\ \text { C } & -0.233194 & -1.207689 & 0.376657 \\ \text { C } & -1.182870 & -0.251844 & 0.097088 \\ \text { C } & -0.227123 & 1.493270 & 0.079160 \\ \text { C } & 1.028091 & 1.336578 & -0.014562 \\ \text { C } & 1.942595 & 0.220767 & 0.017309\end{array}$

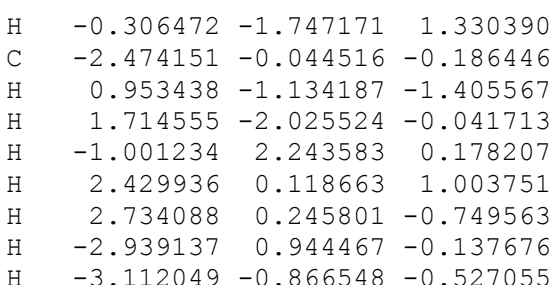

$\mathrm{Ibd}-\mathrm{TS}=\mathrm{Icg}-\mathrm{TS}$

$E(R B L Y P)=271.24964$

$E(\operatorname{RBD}(T))=270.60114$

$\mathrm{ZPE}=74.21$

C $\quad-1.076760-0.271646-0.070389$

C $\quad-0.121431-1.248835-0.298555$

$\begin{array}{llll}1.242411 & -1.161198 & 0.255037\end{array}$

$\begin{array}{lll}1.895362 & 0.313031 & -0.022593\end{array}$

$1.065960 \quad 1.318251-0.034006$

$-0.327699 \quad 1.454950-0.029655$

$-0.289382-1.937634-1.135165$

$\begin{array}{lll}1.271922-1.237129 & 1.356447\end{array}$

$1.908496-1.934916-0.159387$

$\begin{array}{lll}-2.407123 & -0.186503 & 0.139051\end{array}$

$2.9810650 .313358-0.136508$

$-0.783437 \quad 1.861997-0.947094$

$\begin{array}{lll}-0.774612 & 1.918093 & 0.862650\end{array}$

$\begin{array}{lll}-2.960222 & 0.749224 & 0.011273\end{array}$

$\begin{array}{lll}-2.978151 & -1.041297 & 0.514446\end{array}$

Ibe-TS = Igh-TS

$\mathrm{E}(\mathrm{RBLYP})=270.02309$

$E(\operatorname{RBD}(T))=269.37238$

$\mathrm{ZPE}=59.52$

$\begin{array}{lrrr}\text { C } & -1.147355 & -1.220537 & -0.000560 \\ \mathrm{C} & 0.285590 & -1.325185 & 0.001053 \\ \mathrm{C} & 1.045255 & 0.183313 & 0.000021 \\ \mathrm{C} & 0.161429 & 1.276622 & 0.000624 \\ \mathrm{C} & -1.103570 & 1.434813 & 0.000358 \\ \mathrm{C} & -2.025847 & -0.309549 & -0.000878 \\ \mathrm{H} & -1.876492 & 2.191921 & 0.000349 \\ \mathrm{H} & -3.088437 & -0.109067 & -0.001153 \\ \mathrm{C} & 2.397925 & 0.172272 & -0.000910 \\ \mathrm{H} & 0.673488 & -1.843981 & 0.894805 \\ \mathrm{H} & 0.675756 & -1.846160 & -0.890407 \\ \mathrm{H} & 2.971780 & 1.100461 & -0.000233 \\ \mathrm{H} & 2.963345 & -0.763662 & -0.001611\end{array}$

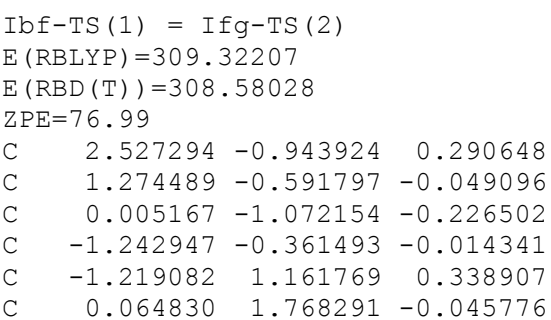




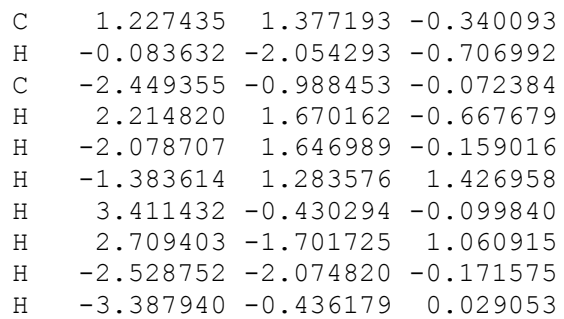

Ibg-TS

$\mathrm{E}(\mathrm{RBLYP})=231.95495$

$\mathrm{E}(\mathrm{RBD}(\mathrm{T}))=231.39821$

$\mathrm{ZPE}=56.85$

$\begin{array}{lrrr}\text { C } & -0.350407 & 1.354074 & -0.000015 \\ \text { C } & -1.479864 & 0.486164 & 0.000203 \\ \text { C } & -0.931850 & -1.147441 & -0.000272 \\ \text { C } & 0.345394 & -1.349024 & 0.000126 \\ \text { C } & 1.490455 & -0.529089 & 0.000146 \\ \text { C } & 0.923461 & 1.189022 & -0.000070 \\ \text { H } & 1.792549 & 1.842286 & -0.000852 \\ \text { H } & -2.119634 & 0.566455 & 0.897301 \\ \text { H } & -2.119839 & 0.566521 & -0.896746 \\ \text { H } & -1.779620 & -1.831754 & -0.000595 \\ \text { H } & 2.121783 & -0.582897 & 0.903319 \\ \text { H } & 2.121624 & -0.582857 & -0.903139\end{array}$

Ibh-TS $=$ Ieg-TS

$\mathrm{E}(\mathrm{RBLYP})=270.02825$

$\mathrm{E}(\mathrm{RBD}(\mathrm{T}))=269.37320$

$\mathrm{ZPE}=59.76$

$\begin{array}{lrrr}\text { C } & 1.051597 & 1.268927 & 0.000004 \\ \text { C } & 1.954429 & 0.148503 & 0.000194 \\ \text { C } & 1.103568 & -1.257127 & 0.000087 \\ \text { C } & -0.208931 & -1.241752 & -0.000668 \\ \text { C } & -1.171724 & -0.288828 & 0.000016 \\ \text { C } & -0.203859 & 1.441829 & -0.000248 \\ \text { H } & -0.977858 & 2.198096 & -0.000229 \\ \text { H } & 2.610143 & 0.112959 & -0.890245 \\ \text { H } & 2.609685 & 0.112922 & 0.890973 \\ \text { H } & 1.754124 & -2.133528 & 0.000542 \\ \text { C } & -2.501827 & -0.107279 & 0.000480 \\ \text { H } & -2.953709 & 0.887035 & -0.000352 \\ \text { H } & -3.181904 & -0.963116 & 0.000123\end{array}$

$\operatorname{ICC}-T S(1)=\operatorname{Idd}-\mathrm{TS}(2)$

$\mathrm{E}(\mathrm{RBLYP})=310.54512$

$E(\operatorname{RBD}(T))=309.80616$

$\mathrm{ZPE}=91.85$

$\begin{array}{llll}\text { C } & -0.605668 & -1.421222 & 0.330550\end{array}$

$\begin{array}{llll}\text { C } & -0.605681 & 1.421223 & -0.330535\end{array}$

C $\quad-1.845115-0.782275-0.191668$

$\begin{array}{llll}\text { C } & 0.664430 & -1.010049 & 0.023728\end{array}$

$\begin{array}{llll}\text { C } & -1.845132 & 0.782259 & 0.191657\end{array}$

C $\quad 0.664420 \quad 1.010053-0.023721$

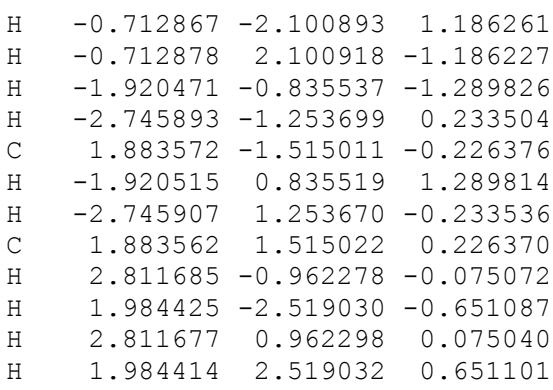

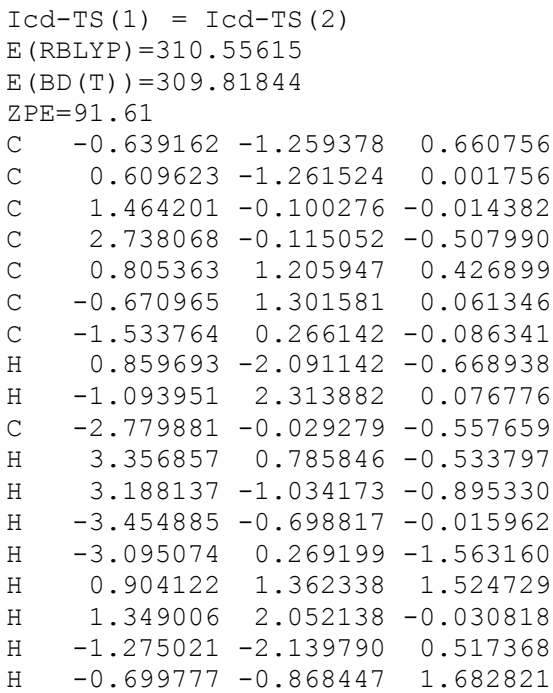

Ice-TS $=$ Idh-TS

$\mathrm{E}(\mathrm{RBLYP})=309.32368$

$\mathrm{E}(\mathrm{RBD}(\mathrm{T}))=308.58240$

$\mathrm{ZPE}=77.15$

$\begin{array}{lrrr}\text { C } & -0.661462 & -1.195180 & 0.328395 \\ \mathrm{C} & 0.650169 & -1.210364 & -0.360337 \\ \mathrm{C} & 1.578983 & -0.231216 & -0.112020 \\ \mathrm{C} & 0.578031 & 1.475272 & -0.061646 \\ \mathrm{C} & -0.681443 & 1.302062 & 0.048485 \\ \mathrm{C} & -1.516939 & 0.160293 & 0.057806 \\ \mathrm{H} & 0.743200 & -1.793095 & -1.285670 \\ \mathrm{C} & 2.871796 & 0.016228 & 0.136574 \\ \mathrm{H} & 1.328540 & 2.249274 & -0.161105 \\ \mathrm{C} & -2.859795 & 0.088195 & -0.085599 \\ \mathrm{H} & -3.469122 & 0.990283 & -0.170003 \\ \mathrm{H} & -3.381353 & -0.871887 & -0.110815 \\ \mathrm{H} & 3.312158 & 1.012823 & 0.039054 \\ \mathrm{H} & 3.532777 & -0.774609 & 0.505374 \\ \mathrm{H} & -1.287628 & -2.055090 & 0.047372 \\ \mathrm{H} & -0.534603 & -1.189447 & 1.425843\end{array}$

$\operatorname{ICf}-T S(1)=\operatorname{Idf}-T S(2)$

$E(R B L Y P)=348.62469$

$\mathrm{E}(\operatorname{RBD}(\mathrm{T}))=347.78958$

$\mathrm{ZPE}=94.14$

$\begin{array}{lrrr}\text { C } & 1.222191 & -0.881009 & -0.147134 \\ \text { C } & 0.210818 & -1.564484 & 0.424694 \\ \text { C } & -1.217115 & -1.111413 & 0.649276 \\ \text { C } & -1.608170 & 0.174802 & -0.065837 \\ \text { C } & -2.882840 & 0.363507 & -0.506712 \\ \text { C } & -0.581889 & 1.180610 & -0.269111\end{array}$ 


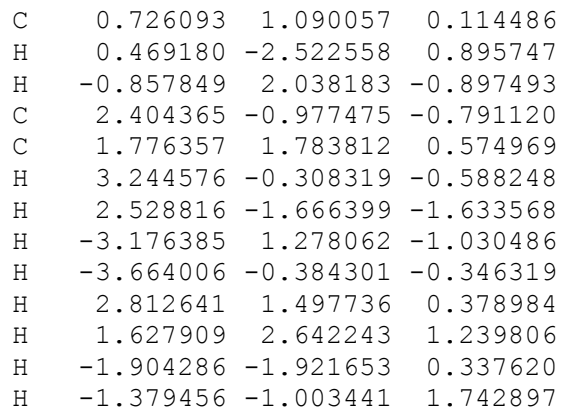

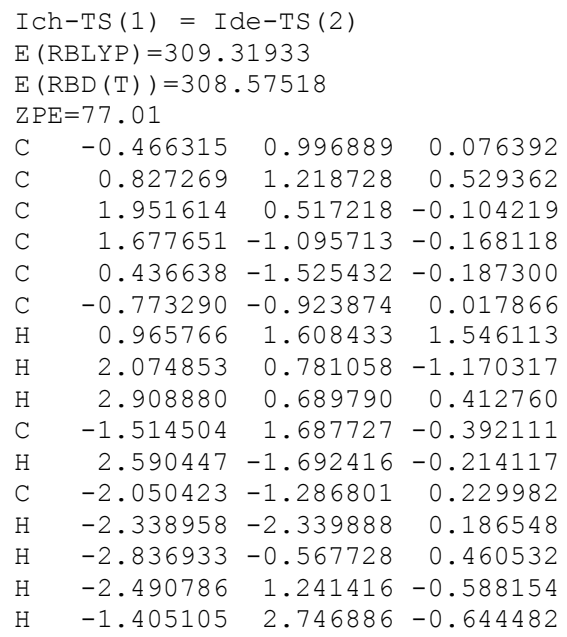

$\operatorname{Idd}-\mathrm{TS}(1)=\operatorname{ICC}-\mathrm{TS}(2)$

$E(R B L Y P)=310.56469$

$\mathrm{E}(\mathrm{RBD}(\mathrm{T}))=309.82989$

$\mathrm{ZPE}=92.55$

$\begin{array}{lrrr}\mathrm{C} & -1.795459 & -0.795502 & 0.550015 \\ \mathrm{C} & -0.597907 & -1.436945 & 0.116095 \\ \mathrm{C} & 0.669040 & -0.760688 & -0.030680 \\ \mathrm{C} & 1.828896 & -1.450294 & -0.274542 \\ \mathrm{C} & 0.668444 & 0.760859 & 0.030363 \\ \mathrm{C} & 1.827738 & 1.451195 & 0.275216 \\ \mathrm{C} & -0.598395 & 1.436842 & -0.118868 \\ \mathrm{C} & -1.797303 & 0.794356 & -0.548078 \\ \mathrm{H} & -0.685775 & -2.417156 & -0.365501 \\ \mathrm{H} & -0.685660 & 2.418280 & 0.360385 \\ \mathrm{H} & 2.767872 & -0.946449 & -0.508164 \\ \mathrm{H} & 1.845752 & -2.543687 & -0.258684 \\ \mathrm{H} & 1.844334 & 2.544565 & 0.257797 \\ \mathrm{H} & 2.766596 & 0.947963 & 0.510569 \\ \mathrm{H} & -2.724922 & 1.362857 & -0.388254 \\ \mathrm{H} & -1.820040 & 0.255981 & -1.498938 \\ \mathrm{H} & -2.723663 & -1.363552 & 0.392358 \\ \mathrm{H} & -1.814819 & -0.257738 & 1.501300\end{array}$

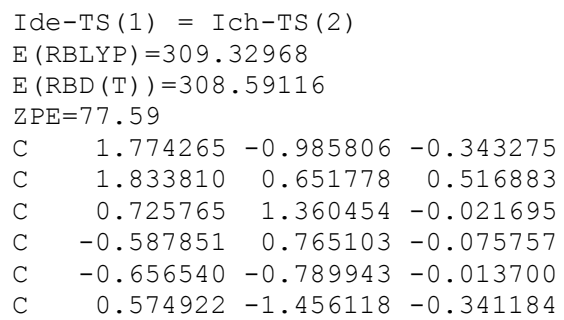

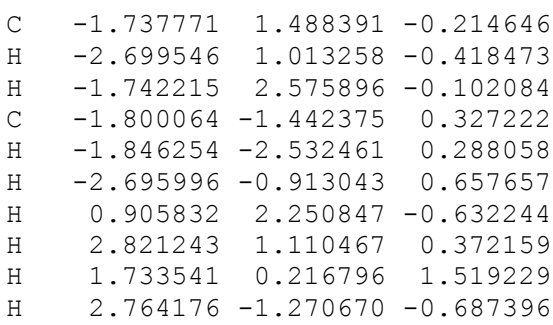

\begin{tabular}{|c|c|c|}
\hline $\begin{array}{l}\operatorname{Idf}-T S(1)=I \\
\mathrm{E}(\operatorname{RBLYP})=348 . \\
\mathrm{E}(\operatorname{RBD}(\mathrm{T}))=347 \\
\mathrm{ZPE}=94.80\end{array}$ & $\begin{array}{l}64062 \\
.80722\end{array}$ & \\
\hline-1.663883 & 0.404748 & -0.110164 \\
\hline-0.567160 & 1.222546 & -0.055927 \\
\hline 0.834374 & 0.826366 & 0.094623 \\
\hline 1.788414 & 1.772547 & 0.330237 \\
\hline 1.181263 & -0.628527 & -0.090741 \\
\hline 2.410811 & -1.025171 & -0.533278 \\
\hline 0.089349 & -1.570986 & 0.077273 \\
\hline-1.071502 & -1.268654 & 0.803143 \\
\hline-0.761594 & 2.298913 & 0.035763 \\
\hline 0.108247 & -2.476950 & -0.538353 \\
\hline-2.949570 & 0.337181 & -0.514276 \\
\hline-1.003390 & -0.745802 & 1.760550 \\
\hline-1.894090 & -1.990484 & 0.762274 \\
\hline-3.708256 & -0.225139 & 0.037712 \\
\hline-3.263638 & 0.767674 & -1.472164 \\
\hline 2.836740 & 1.512937 & 0.488039 \\
\hline 1.532457 & 2.834837 & 0.374925 \\
\hline 2.629940 & -2.082491 & -0.707894 \\
\hline 3.211001 & -0.313796 & -0.746198 \\
\hline
\end{tabular}

Iee-TS1 = Ihh-TS2

$\mathrm{E}(\mathrm{RBLYP})=308.09826$

$E(R B D(T))=307.35410$

$\mathrm{ZPE}=62.77$

$\begin{array}{lrrr}\text { C } & 0.687329 & 1.389313 & -0.221596 \\ \mathrm{C} & -0.584290 & 0.801469 & 0.005813 \\ \mathrm{C} & -0.584485 & -0.801415 & -0.005865 \\ \mathrm{C} & 0.687076 & -1.389448 & 0.221463 \\ \mathrm{C} & 1.883641 & -0.951286 & 0.205832 \\ \mathrm{C} & 1.883841 & 0.951018 & -0.205811 \\ \mathrm{H} & 2.917565 & -1.245962 & 0.327939 \\ \mathrm{H} & 2.917832 & 1.245592 & -0.327583 \\ \mathrm{C} & -1.735417 & -1.475090 & -0.266915 \\ \mathrm{C} & -1.735033 & 1.475378 & 0.267024 \\ \mathrm{H} & -1.772763 & -2.563249 & -0.196844 \\ \mathrm{H} & -2.653325 & -0.960855 & -0.555586 \\ \mathrm{H} & -2.653070 & 0.961313 & 0.555594 \\ \mathrm{H} & -1.772211 & 2.563531 & 0.196814\end{array}$

Ief-TS $(1)=\operatorname{Ifh}-\mathrm{TS}(2)$

$E(R B L Y P)=347.40655$

$\mathrm{E}(\operatorname{RBD}(\mathrm{T}))=346.56441$

$\mathrm{ZPE}=80.18$

$\begin{array}{lrrr}\text { C } & 0.681698 & 1.013244 & -0.080725 \\ \text { C } & -0.675785 & 1.114942 & -0.138271 \\ \text { C } & -1.668824 & 0.050593 & -0.002223 \\ \text { C } & -1.188398 & -1.349719 & -0.040653 \\ \text { C } & 0.129569 & -1.567942 & -0.028071 \\ \text { C } & 1.263984 & -0.838245 & -0.014825\end{array}$ 


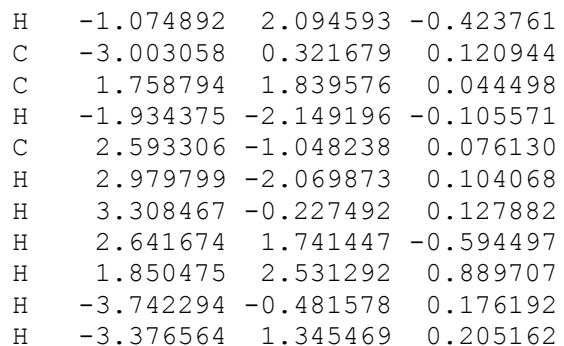

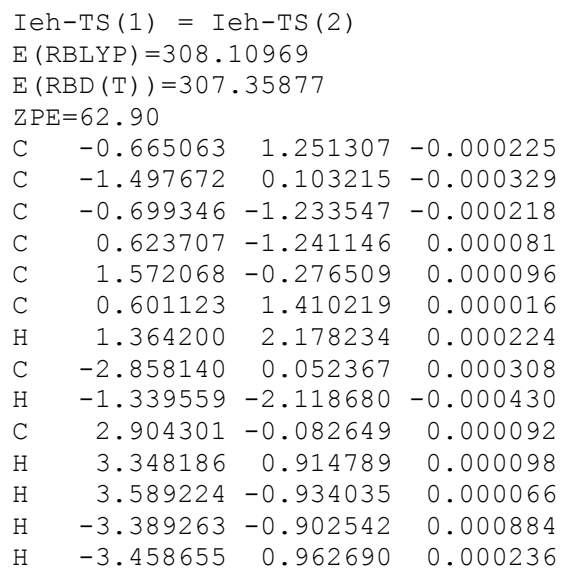

$\operatorname{Iff-TS}(1)=\operatorname{Iff-TS}(2)$

$E($ RBLYP $)=386.70868$

$\mathrm{E}(\operatorname{RBD}(\mathrm{T}))=385.77863$

$\mathrm{ZPE}=97.23$

$\begin{array}{llll}\text { C } & -1.217196 & -1.012716 & 0.277010\end{array}$

C $\quad-0.009722-1.459192-0.159341$

C $\quad 1.243833-0.712742-0.231264$

C $\quad 2.365061-1.312213-0.722642$

$\begin{array}{llll}\text { C } & 1.243431 & 0.713113 & 0.231111\end{array}$

$\begin{array}{llll}\text { C } & 2.363914 & 1.312915 & 0.723722\end{array}$

$\begin{array}{llll}\text { C } & -0.009968 & 1.459687 & 0.156769\end{array}$

C $\quad-1.217900 \quad 1.012233-0.277211$

$0.009231-2.462160-0.606142$

$\begin{array}{lll}0.009456 & 2.464047 & 0.600407\end{array}$

$\begin{array}{lll}-2.372103 & -1.319225 & 0.882446\end{array}$

$\begin{array}{lll}-2.373962 & 1.318105 & -0.880956\end{array}$

$\begin{array}{lll}-3.323516 & -0.844644 & 0.627915\end{array}$

$\begin{array}{lll}-2.389401 & -2.010009 & 1.733373\end{array}$

$2.349664-2.355791-1.049334$

$\begin{array}{lll}2.348085 & 2.356745 & 1.049580\end{array}$

$\begin{array}{lll}3.310313 & 0.779164 & 0.826268\end{array}$

$\begin{array}{llll}-3.325219 & 0.845047 & -0.622972\end{array}$

$\begin{array}{lll}-2.392652 & 2.006318 & -1.733934\end{array}$
$3.311715-0.778508-0.823031$

$$
\begin{array}{rrr}
0.184603 & -2.047614 & -0.661139 \\
2.527277 & -0.918516 & 0.119147 \\
-2.451052 & -1.072117 & 0.124573 \\
2.168877 & 1.746695 & -0.045089 \\
-1.893866 & 1.560445 & -0.891062 \\
-1.881151 & 1.482590 & 0.923087 \\
-3.343886 & -0.576376 & -0.270195 \\
-2.625582 & -1.908069 & 0.810760 \\
3.449401 & -0.337530 & 0.204290 \\
2.618716 & -2.004968 & 0.195845
\end{array}
$$

$\operatorname{Ifh}-\mathrm{TS}(1)=\operatorname{Ief}-\mathrm{TS}(2)$

$\mathrm{E}(\mathrm{RBLYP})=347.40172$

$\mathrm{E}(\operatorname{RBD}(\mathrm{T}))=346.56361$ $\mathrm{ZPE}=79.96$

$\begin{array}{lrrr}\text { C } & 1.134892 & -1.478019 & -0.493614 \\ \text { C } & -0.115772 & -1.561951 & -0.308835 \\ \text { C } & -1.185499 & -0.672550 & 0.048528 \\ \text { C } & -2.386270 & -1.100395 & 0.520119 \\ \text { C } & -0.819803 & 0.794643 & -0.110735 \\ \text { C } & -1.787802 & 1.748626 & -0.230158 \\ \text { C } & 0.591921 & 1.174468 & -0.120864 \\ \mathrm{C} & 1.676189 & 0.363256 & 0.040813 \\ \text { H } & 0.798441 & 2.211359 & -0.411513 \\ \mathrm{C} & 2.951781 & 0.320901 & 0.472080 \\ \text { H } & -3.155264 & -0.402719 & 0.857814 \\ \mathrm{H} & -2.605886 & -2.167357 & 0.594952 \\ \mathrm{H} & -1.540936 & 2.813657 & -0.211183 \\ \mathrm{H} & -2.844371 & 1.491459 & -0.325181 \\ \mathrm{H} & 3.708006 & -0.322294 & 0.010577 \\ \mathrm{H} & 3.254872 & 0.843069 & 1.386662 \\ \mathrm{H} & 2.027312 & -2.001053 & -0.806140\end{array}$

Ihh-TS1 $=$ Iee-TS2

$\mathrm{E}(\mathrm{RBLYP})=308.10231$

$\mathrm{E}(\operatorname{RBD}(\mathrm{T}))=307.35175$

$\mathrm{ZPE}=62.92$

$\begin{array}{lrrr}\text { C } & -0.561069 & -0.932746 & 0.014318 \\ \text { C } & 0.714318 & -1.388641 & 0.235083 \\ \text { C } & 1.878372 & -0.766285 & 0.150275 \\ \text { C } & 1.879077 & 0.765118 & -0.149741 \\ \text { C } & 0.715506 & 1.388165 & -0.236161 \\ \text { C } & -0.559988 & 0.933037 & -0.014239 \\ \mathrm{C} & -1.760144 & -1.503544 & -0.241945 \\ \text { H } & 2.872357 & 1.204341 & -0.249083 \\ \mathrm{C} & -1.758595 & 1.504711 & 0.242162 \\ \mathrm{H} & -1.890800 & 2.582203 & 0.122529 \\ \mathrm{H} & -2.621657 & 0.927617 & 0.574084 \\ \mathrm{H} & -2.622983 & -0.925716 & -0.573214 \\ \mathrm{H} & -1.893041 & -2.580995 & -0.122746 \\ \mathrm{H} & 2.871253 & -1.206342 & 0.249924\end{array}$

$\operatorname{Ifg}-\mathrm{TS}(1)=\operatorname{Ibf}-\mathrm{TS}(2)$

$\mathrm{E}(\mathrm{RBLYP})=309.33278$

$E(B D(T))=308.58789$

$\mathrm{ZPE}=77.26$

$\begin{array}{llll}\text { C } & -1.201519 & -0.575313 & -0.081727\end{array}$

C $\quad 0.077641-1.048865-0.222919$

C $\quad 1.315689-0.306962-0.017508$

$\begin{array}{llll}\text { C } & 1.224241 & 1.193628 & 0.000119\end{array}$

$\begin{array}{llll}\text { C } & 0.026688 & 1.752108 & 0.027398\end{array}$

$\begin{array}{llll}\text { C } & -1.298484 & 1.323509 & 0.006501\end{array}$
$\mathrm{IaC}=\mathrm{Iad}$

$E($ UBLYP $)=272.49264$

$\mathrm{E}(\mathrm{UBD}(\mathrm{T}))=271.86126$

$\mathrm{ZPE}=89.05$

$<\mathrm{S} 2, \mathrm{UBLYP}>=0.932$

$<\mathrm{S} 2, \mathrm{UHF}>=1.232$

C $\quad 1.058279 \quad 1.280152-0.178881$

C $\quad-1.086805-0.085667-0.003328$

C $\quad-0.288703-1.234930-0.057704$ $\begin{array}{llll}\text { C } & -0.433159 & 1.305868 & -0.044041\end{array}$ 


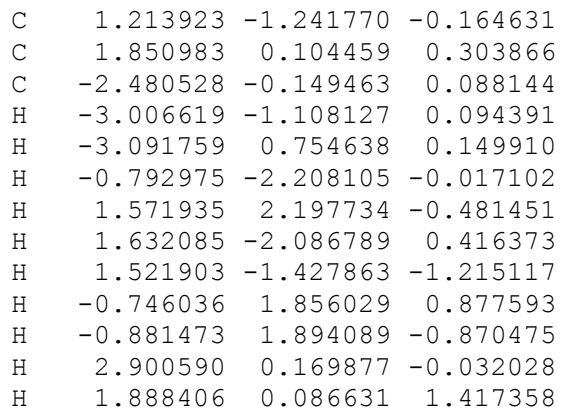

Iaf

$E(\operatorname{UBLYP})=310.58241$

$\mathrm{E}(\mathrm{UBD}(\mathrm{T}))=309.85181$

$\mathrm{ZPE}=92.03$

$<\mathrm{S} 2, \mathrm{UBLYP}>=0.898$

$<\mathrm{S} 2, \mathrm{UHF}>=1.491$

$\begin{array}{lllll}\text { C } & -0.000026 & 1.774308 & -0.200907\end{array}$

C $\quad 1.311514 \quad 1.115680 \quad 0.090491$

C $\quad 1.266865-0.415085 \quad 0.009508$

C $\quad-0.000004-1.064576-0.026932$

$\begin{array}{llll}\text { C } & -1.266883 & -0.415084 & 0.009520\end{array}$

$\begin{array}{lllll}\text { C } & -1.311481 & 1.115669 & 0.090869\end{array}$

C $\quad 2.450120-1.122478-0.018744$

$\mathrm{H} \quad 2.458977-2.214199-0.084756$

H $\quad 3.420675-0.620571 \quad 0.018683$

C $\quad-2.450135-1.122455-0.018969$

$\mathrm{H} \quad-3.420688-0.620549 \quad 0.018508$

$\mathrm{H} \quad-2.458995-2.214161-0.085225$

$\mathrm{H} \quad 0.000000-2.160777-0.062076$

$\mathrm{H} \quad-0.000052 \quad 2.842254-0.441558$

$\mathrm{H} \quad-1.680063 \quad 1.396815 \quad 1.108611$

$\mathrm{H} \quad-2.092779 \quad 1.499206-0.594179$

$\mathrm{H} \quad 2.092533 \quad 1.499010-0.595006$

$\mathrm{H} \quad 1.680577 \quad 1.397095 \quad 1.107983$

$\mathrm{Ibc}=\mathrm{Idg}$

$\mathrm{E}(\mathrm{UBLY})=271.26585$

$E(\operatorname{UBD}(T))=270.62893$

$\mathrm{ZPE}=75.46$

$<\mathrm{S} 2, \mathrm{UBLYP}\rangle=1.015$

$<\mathrm{S} 2, \mathrm{UHF}>=1.582$

$\begin{array}{lrrr}\text { C } & 0.921548 & 1.369210 & -0.038138 \\ \mathrm{C} & -0.404223 & 1.286067 & -0.148280 \\ \mathrm{C} & -1.069243 & -0.053402 & 0.000375 \\ \mathrm{C} & -0.218000 & -1.186207 & 0.058522 \\ \mathrm{C} & 1.259777 & -1.089841 & -0.225670 \\ \mathrm{C} & 1.890107 & 0.277876 & 0.248151 \\ \mathrm{C} & -2.455402 & -0.138824 & 0.084558 \\ \mathrm{H} & -2.960023 & -1.105304 & 0.164401 \\ \mathrm{H} & -3.084815 & 0.754064 & 0.068316 \\ \mathrm{H} & -0.660068 & -2.174869 & 0.217581 \\ \mathrm{H} & 1.810820 & -1.933665 & 0.226589 \\ \mathrm{H} & 1.429192 & -1.162866 & -1.322380 \\ \mathrm{H} & 2.859185 & 0.425705 & -0.260280 \\ \mathrm{H} & 2.105848 & 0.248216 & 1.335033 \\ \mathrm{H} & -1.047520 & 2.159447 & -0.306376\end{array}$

$\mathrm{Ibd}=\mathrm{Icg}$

$E(U B L Y P)=271.26242$

$\mathrm{E}(\mathrm{UBD}(\mathrm{T}))=270.62610$

$\mathrm{ZPE}=75.11$

$<\mathrm{S} 2, \mathrm{UBLYP}>=1.023$

$<\mathrm{S} 2, \mathrm{UHF}>=0.731$

C $\quad-1.033976 \quad 1.334648-0.000133$

$\begin{array}{llll}\text { C } & 0.450006 & 1.343527 & 0.000134\end{array}$

C $\quad 1.018821-0.105826-0.000062$

C $\quad 0.138884-1.195394 \quad-0.000151$

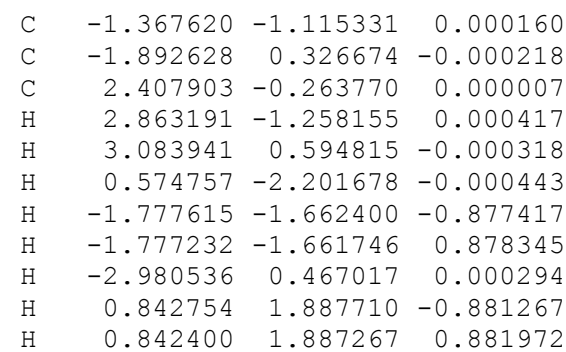

$\mathrm{Ibf}=\mathrm{Ifg}$

$E(U B L Y P)=309.35652$

$\mathrm{E}(\mathrm{UBD}(\mathrm{T}))=308.62279$

$\mathrm{ZPE}=78.44$

$<\mathrm{S} 2, \mathrm{UBLYP}>=1.039$

$<\mathrm{S} 2, \mathrm{UHF}>=1.837$

C $\quad-0.056091 \quad 1.785598-0.000059$

C $\quad-1.245741 \quad 1.188773-0.000189$

C $-1.303128-0.303961-0.000060$

$\begin{array}{llll}\text { C } & -0.041984 & -0.999325 & 0.000007\end{array}$

C $\quad 1.231038-0.369352-0.000020$

$\begin{array}{llll}\text { C } & 1.301935 & 1.186954 & 0.000132\end{array}$

C $\quad-2.509863-0.968096 \quad 0.000053$

$\mathrm{H} \quad-2.557625 \quad-2.060308 \quad 0.000257$

$\mathrm{H} \quad-3.459806-0.428159 \quad 0.000008$

C $\quad 2.411647-1.091632-0.000059$

$\mathrm{H} \quad 3.388466-0.601222-0.000259$

$\mathrm{H} \quad 2.404183-2.185234 \quad 0.000066$

$\mathrm{H} \quad-0.067365-2.094584 \quad 0.000040$

$\mathrm{H} \quad 1.879705 \quad 1.528479-0.881029$

H $\quad 1.879107 \quad 1.528232 \quad 0.881795$

$\mathrm{H} \quad-2.193538 \quad 1.739039 \quad 0.000288$

$I_{C C}=I d d$

$\mathrm{E}(\mathrm{UBLYP})=310.59074$

$\mathrm{E}(\mathrm{UBD}(\mathrm{T}))=309.86378$

$\mathrm{ZPE}=92.63$

$<\mathrm{S} 2, \mathrm{UBLYP}>=0.950$

$<\mathrm{S} 2, \mathrm{UHF}>=1.515$

C $\quad 0.559258 \quad 1.418482 \quad 0.091616$

C $\quad-0.676964 \quad 0.753798 \quad-0.031603$

$\begin{array}{llll}\text { C } & -0.676964 & -0.753797 & 0.031603\end{array}$

C $\quad 0.559258-1.418482-0.091616$

C $\quad 1.867974-0.710604-0.315242$

$\begin{array}{lllll}\text { C } & 1.867974 & 0.710604 & 0.315242\end{array}$

C $\quad-1.860607 \quad 1.475002-0.213398$

$\mathrm{H} \quad-2.828358 \quad 0.996474 \quad-0.362284$

$\mathrm{H} \quad-1.840330 \quad 2.567291-0.250245$

$\begin{array}{llll}\text { C } & -1.860607 & -1.475002 & 0.213398\end{array}$

$\mathrm{H} \quad-1.840330-2.567291 \quad 0.250245$

$\mathrm{H} \quad-2.828358-0.996474 \quad 0.362283$

H $\quad 0.561231-2.513699-0.063234$

$\mathrm{H} \quad 0.561231 \quad 2.513699 \quad 0.063234$

H $\quad 2.705232-1.310973 \quad 0.084334$

$\mathrm{H} \quad 2.064261-0.613894-1.405393$

$\mathrm{H} \quad 2.705232 \quad 1.310973-0.084334$

$\begin{array}{llll}\mathrm{H} & 2.064261 & 0.613894 & 1.405393\end{array}$

Icd

$\mathrm{E}(\mathrm{UBLYP})=310.59007$

$E(\operatorname{UBD}(T))=309.86179$

$\mathrm{ZPE}=92.19$

$<\mathrm{S} 2, \mathrm{UBLYP}>=0.911$

$<\mathrm{S} 2, \mathrm{UHF}>=1.431$

C $\quad 0.725560-1.230253-0.000293$

C $\quad-0.774234-1.301559 \quad 0.002416$

C $\quad-1.482823 \quad 0.055088-0.000125$

C $\quad-0.725560 \quad 1.230253-0.000292$ 


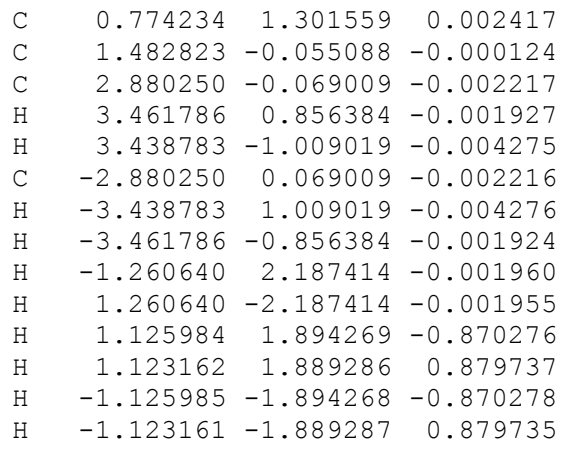

$I c e=I d h$

$E(U B L Y P)=309.34520$

$E(\operatorname{UBD}(T))=308.61221$

$\mathrm{ZPE}=78.03$

$<\mathrm{S} 2, \mathrm{UBLYP}\rangle=1.035$

$<\mathrm{S} 2, \mathrm{UHF}>=1.935$

$\begin{array}{lrrr}\text { C } & -0.695634 & 1.190623 & -0.043430 \\ \mathrm{C} & 0.786942 & 1.223377 & 0.245900 \\ \mathrm{C} & 1.510358 & -0.128644 & 0.016105 \\ \mathrm{C} & 0.631683 & -1.260471 & 0.147009 \\ \mathrm{C} & -0.709264 & -1.282051 & 0.150794 \\ \mathrm{C} & -1.460457 & -0.001484 & -0.034042 \\ \mathrm{C} & -2.846070 & -0.020290 & -0.182190 \\ \mathrm{H} & -3.406031 & -0.958134 & -0.189950 \\ \mathrm{H} & -3.416567 & 0.905187 & -0.294732 \\ \mathrm{C} & 2.834778 & -0.209124 & -0.267480 \\ \mathrm{H} & 3.332219 & -1.175847 & -0.374963 \\ \mathrm{H} & 3.450002 & 0.686784 & -0.389238 \\ \mathrm{H} & -1.208184 & 2.148296 & -0.178644 \\ \mathrm{H} & 1.286162 & 2.017585 & -0.339672 \\ \mathrm{H} & 0.936468 & 1.510612 & 1.312430 \\ \mathrm{H} & -1.288088 & -2.206100 & 0.258780\end{array}$

$\operatorname{Icf}=\operatorname{Idf}$

$E(U B L Y P)=348.68137$

$E(\operatorname{UBD}(T))=347.85616$

$\mathrm{ZPE}=95.53$

$<\mathrm{S} 2, \mathrm{UBLYP}>=0.888$

$<\mathrm{S} 2, \mathrm{UHF}>=1.772$

C $\quad 0.250751-1.575844-0.000064$

$\begin{array}{llll}\text { C } & 1.237008 & -0.574726 & 0.000001\end{array}$

C $\quad 0.787805 \quad 0.860594 \quad 0.000031$

C $\quad-0.621181 \quad 1.115690-0.000020$

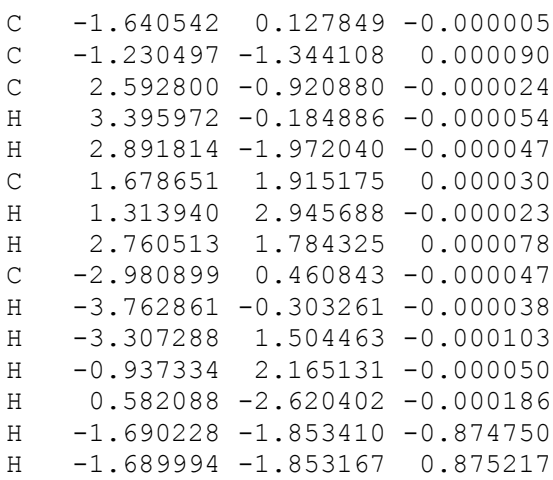

Ich

$E(U B L Y P)=309.34324$

$\mathrm{E}(\mathrm{UBD}(\mathrm{T}))=308.61108$

$\mathrm{ZPE}=77.98$

$<$ S2, UBLYP $>=1.027$

$<\mathrm{S} 2, \mathrm{UHF}>=1.909$

$\begin{array}{lrrr}\text { C } & 0.677605 & 1.315751 & 0.001324 \\ \mathrm{C} & -0.605001 & 0.738618 & -0.000598 \\ \mathrm{C} & -0.712795 & -0.777393 & -0.000208 \\ \mathrm{C} & 0.569216 & -1.447311 & -0.003119 \\ \mathrm{C} & 1.804719 & -0.950099 & -0.002418 \\ \mathrm{C} & 1.984628 & 0.568177 & 0.002724 \\ \mathrm{C} & -1.749744 & 1.550192 & -0.002506 \\ \mathrm{H} & -2.761792 & 1.145781 & -0.005631 \\ \mathrm{H} & -1.653137 & 2.638839 & -0.001264 \\ \mathrm{C} & -1.883600 & -1.468447 & 0.003151 \\ \mathrm{H} & -1.879307 & -2.560356 & 0.003073 \\ \mathrm{H} & -2.859719 & -0.980562 & 0.006365 \\ \mathrm{H} & 0.742237 & 2.409580 & 0.001506 \\ \mathrm{H} & 2.600792 & 0.875339 & -0.871872 \\ \mathrm{H} & 2.595992 & 0.869810 & 0.882691 \\ \mathrm{H} & 2.704751 & -1.575357 & -0.004963\end{array}$

Iee $=$ Ihh

$E($ UBLYP $)=308.10427$

$E(\operatorname{UBD}(T))=307.36056$

$\mathrm{ZPE}=63.74$

$<\mathrm{S} 2, \mathrm{UBLYP}>=0.628$

$<\mathrm{S} 2, \mathrm{UHF}>=1.456$

$\begin{array}{lrrr}\text { C } & -0.699435 & -1.369685 & 0.236869 \\ \mathrm{C} & 0.583394 & -0.795977 & 0.005682 \\ \mathrm{C} & 0.583394 & 0.795977 & -0.005683 \\ \mathrm{C} & -0.699435 & 1.369685 & -0.236869 \\ \mathrm{C} & -1.874635 & 0.749080 & -0.169434 \\ \mathrm{C} & -1.874635 & -0.749080 & 0.169435 \\ \mathrm{C} & 1.731268 & -1.486742 & -0.246205 \\ \mathrm{H} & 2.652876 & -0.986364 & -0.547552 \\ \mathrm{H} & 1.758705 & -2.573505 & -0.148355 \\ \mathrm{C} & 1.731268 & 1.486743 & 0.246205 \\ \mathrm{H} & 1.758704 & 2.573505 & 0.148356 \\ \mathrm{H} & 2.652875 & 0.986365 & 0.547553 \\ \mathrm{H} & -2.855133 & 1.207621 & -0.317041 \\ \mathrm{H} & -2.855133 & -1.207622 & 0.317038\end{array}$

Ief $=$ Ifh

$E(U B L Y P)=347.43870$

$\mathrm{E}(\operatorname{UBD}(\mathrm{T}))=346.60864$

$\mathrm{ZPE}=81.41$

$<\mathrm{S} 2, \mathrm{UBLYP}>=1.054$

$<\mathrm{S} 2, \mathrm{UHF}>=2.284$

C $\quad 0.146320-1.587891-0.000034$

$\begin{array}{llll}\text { C } & 1.236136 & -0.641979 & 0.000047\end{array}$

C $\quad 0.779342 \quad 0.805927-0.000070$

C $\quad-0.622655 \quad 1.061800-0.000026$ 


$\begin{array}{lrrr}\text { C } & -1.643714 & 0.047885 & 0.000028 \\ \text { C } & -1.174538 & -1.361774 & -0.000219 \\ \text { C } & 2.537150 & -1.048096 & 0.000119 \\ \text { H } & 3.375171 & -0.348890 & 0.000087 \\ \text { H } & 2.782238 & -2.111976 & 0.000159 \\ \text { C } & 1.683610 & 1.862949 & -0.000053 \\ \text { H } & 1.325262 & 2.895467 & -0.000056 \\ \text { H } & 2.764428 & 1.718905 & -0.000011 \\ \mathrm{C} & -2.989603 & 0.351908 & 0.000126 \\ \mathrm{H} & -3.751974 & -0.430718 & 0.000095 \\ \mathrm{H} & -3.338485 & 1.387882 & 0.000163 \\ \mathrm{H} & -0.947155 & 2.107455 & 0.000030 \\ \mathrm{H} & -1.921771 & -2.162499 & 0.000030\end{array}$

Ieh

$\mathrm{E}(\mathrm{UBLYP})=308.11587$

$E(\operatorname{UBD}(T))=307.37020$

$\mathrm{ZPE}=63.90$

$\langle\mathrm{S} 2, \mathrm{UBLYP}\rangle=0.630$

$<\mathrm{S} 2, \mathrm{UHF}>=1.155$

$\begin{array}{llll}\text { C } & -0.612197 & 1.237598 & -0.000106\end{array}$

C $\quad 0.715284 \quad 1.229857-0.000020$

$\begin{array}{llll}\text { C } & 1.484149 & -0.110205 & 0.000019\end{array}$

$\begin{array}{llll}\text { C } & 0.612197 & -1.237598 & 0.000090\end{array}$

C $\quad-0.715284-1.229857-0.000109$

$\begin{array}{llll}\text { C } & -1.484149 & 0.110206 & 0.000010\end{array}$

$\begin{array}{llll}\text { C } & -2.848092 & 0.109630 & 0.000047\end{array}$

$\mathrm{H} \quad-3.415611-0.823814 \quad 0.000010$

$\begin{array}{llll}\mathrm{H} & -3.412631 & 1.043163 & 0.000111\end{array}$

C $\quad 2.848093-0.109630-0.000003$

$\mathrm{H} \quad 3.412629-1.043165-0.000033$

$\mathrm{H} \quad \begin{array}{llll}.415613 & 0.823812 & -0.000074\end{array}$

$\mathrm{H} \quad-1.355042 \quad-2.117006 \quad 0.000044$

$\begin{array}{llll}\mathrm{H} & 1.355041 & 2.117007 & 0.000370\end{array}$
Iff

$E($ UBLYP $)=386.77380$

$\mathrm{E}(\mathrm{UBD}(\mathrm{T}))=385.85185$

$\mathrm{ZPE}=98.85$

$<\mathrm{S} 2, \mathrm{UBLYP}\rangle=0.883$

$<\mathrm{S} 2, \mathrm{UHF}\rangle=2.114$

C $\quad-0.000001 \quad 1.401901-0.000885$

$\begin{array}{llll}\text { C } & -1.269605 & 0.749925 & -0.000459\end{array}$

$\begin{array}{llll}\text { C } & -1.269604 & -0.749927 & 0.000488\end{array}$

$\begin{array}{llll}\text { C } & 0.000000 & -1.401902 & 0.000896\end{array}$

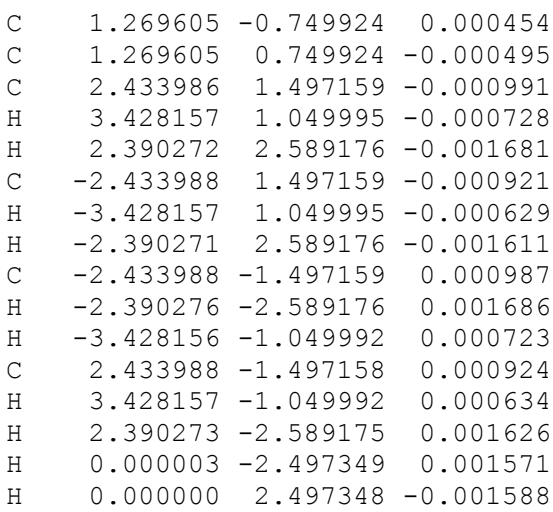




\section{Branch-II}

$S(I I)$ aa

$E($ RBLYP $)=233.27025$

$\mathrm{E}(\operatorname{RBD}(\mathrm{T}))=232.17982$

$\mathrm{ZPE}=72.69$

$\begin{array}{lrrr}\text { C } & 0.000000 & 2.919447 & -0.319002 \\ \text { C } & 0.000000 & 1.566115 & -0.400533 \\ \text { C } & 0.000000 & 0.685016 & 0.757358 \\ \text { C } & 0.000000 & -0.685016 & 0.757358 \\ \text { C } & 0.000000 & -1.566115 & -0.400533 \\ \text { C } & 0.000000 & -2.919447 & -0.319002 \\ \text { H } & 0.000000 & 1.189843 & 1.731457 \\ \text { H } & 0.000000 & -1.189843 & 1.731457 \\ \text { H } & 0.000000 & 1.103151 & -1.394025 \\ \text { H } & 0.000000 & 3.550209 & -1.211418 \\ \text { H } & 0.000000 & 3.435192 & 0.647043 \\ \text { H } & 0.000000 & -1.103151 & -1.394025 \\ \text { H } & 0.000000 & -3.550209 & -1.211418 \\ \text { H } & 0.000000 & -3.435192 & 0.647043\end{array}$

$S(I I) a b$

$E($ RBLYP $)=232.02508$

$E(R B D(T))=231.47097$

$\mathrm{ZPE}=57.82$

$\begin{array}{lrrr}\mathrm{C} & 1.381677 & 1.380126 & -0.000233 \\ \mathrm{C} & 1.714270 & 0.065768 & 0.000251 \\ \mathrm{C} & 0.826889 & -1.098952 & 0.000012 \\ \mathrm{C} & -0.541458 & -1.173486 & -0.000218 \\ \mathrm{C} & -1.471694 & -0.101535 & 0.000480 \\ \mathrm{C} & -2.337959 & 0.763940 & -0.000437 \\ \mathrm{H} & 2.781663 & -0.188981 & 0.000749 \\ \mathrm{H} & 1.343759 & -2.065377 & -0.000208 \\ \mathrm{H} & -0.988453 & -2.175578 & -0.000552 \\ \mathrm{H} & -3.074091 & 1.543406 & 0.001706 \\ \mathrm{H} & 2.160577 & 2.147740 & -0.000071 \\ \mathrm{H} & 0.346197 & 1.723621 & -0.000756\end{array}$

$S(I I)$ ac

$E($ RBLYP $)=271.32862$

$\mathrm{E}(\mathrm{RBD}(\mathrm{T}))=270.67909$

$\mathrm{ZPE}=75.18$

$\begin{array}{lrrr}\mathrm{C} & -2.106002 & -0.413773 & 0.000003 \\ \mathrm{C} & -1.254386 & 0.765914 & 0.000003 \\ \mathrm{C} & 0.114322 & 0.801577 & -0.000017 \\ \mathrm{C} & 1.016083 & -0.348656 & -0.000071 \\ \mathrm{C} & 2.342732 & -0.252185 & 0.000052 \\ \mathrm{C} & 3.652542 & -0.155746 & 0.000027 \\ \mathrm{C} & -3.460954 & -0.369860 & 0.000035 \\ \mathrm{H} & 4.231584 & -0.113318 & 0.931404 \\ \mathrm{H} & 4.231522 & -0.113168 & -0.931383 \\ \mathrm{H} & -1.616958 & -1.395230 & -0.000063 \\ \mathrm{H} & 0.575795 & -1.354063 & -0.000166 \\ \mathrm{H} & -1.780573 & 1.728526 & -0.000012 \\ \mathrm{H} & 0.602622 & 1.781730 & -0.000026 \\ \mathrm{H} & -4.066536 & -1.279536 & 0.000017 \\ \mathrm{H} & -4.003477 & 0.581436 & 0.000046\end{array}$

$S(I I) b b$

$\mathrm{E}(\mathrm{RBLYP})=230.78812$

$E(\operatorname{RBD}(T)))=230.22929$

$\mathrm{ZPE}=42.70$

C $\quad 0.685238 \quad 1.078274-0.000015$

C $\quad-0.685239 \quad 1.078274 \quad-0.000015$

$\mathrm{H} \quad 1.193889 \quad 2.049528-0.000015$

$\mathrm{H} \quad-1.193889 \quad 2.049528-0.000015$

C $\quad 1.512764-0.073748-0.000015$

C $\quad-1.512764-0.073748-0.000015$

C $\quad 2.273868-1.030953-0.000015$

C $\quad-2.273869-1.030953-0.000015$

$\mathrm{H} \quad 2.914093-1.890964 \quad 0.000581$

$\mathrm{H} \quad-2.914089-1.890967 \quad-0.000015$

$\mathrm{S}$ (II) bc (s-trans)

$E(R B L Y P)=270.09002$

$E(\operatorname{RBD}(T))=269.43629$

$\mathrm{ZPE}=60.24$

$\begin{array}{lrrr}\text { C } & -3.052787 & -1.023622 & 0.000003 \\ \text { C } & -2.266726 & -0.085628 & 0.000011 \\ \text { C } & -1.392647 & 1.034756 & -0.000007 \\ \text { C } & -0.024661 & 0.971010 & -0.000011 \\ \text { C } & 0.751625 & -0.263348 & -0.000023 \\ \mathrm{C} & 2.080714 & -0.306855 & -0.000001 \\ \mathrm{C} & 3.393097 & -0.349334 & 0.000014 \\ \mathrm{H} & 3.972373 & -0.368958 & 0.931726 \\ \mathrm{H} & 3.972386 & -0.369028 & -0.931689 \\ \mathrm{H} & 0.186431 & -1.204905 & -0.000019 \\ \mathrm{H} & 0.535709 & 1.910902 & 0.000009 \\ \mathrm{H} & -1.866681 & 2.023242 & 0.000034 \\ \mathrm{H} & -3.731903 & -1.853126 & 0.000022\end{array}$

$\mathrm{S}(\mathrm{II}) \mathrm{bc} \quad(\mathrm{S}-\mathrm{Cis})$

$\mathrm{E}(\mathrm{RBLYP})=270.08355$

$E(\operatorname{RBD}(\mathrm{T})))=269.43012$

$\mathrm{ZPE}=60.14$

$\begin{array}{lrrr}\text { C } & 2.146936 & 1.250222 & 0.000020 \\ \text { C } & 1.581722 & 0.065559 & -0.000018 \\ \text { C } & 1.089952 & -1.170847 & -0.000054 \\ \mathrm{C} & -0.308350 & -1.609122 & -0.000007 \\ \mathrm{C} & -1.461477 & -0.871549 & 0.000002 \\ \mathrm{C} & -1.579741 & 0.542120 & -0.000024 \\ \mathrm{C} & -1.778326 & 1.749773 & 0.000021 \\ \mathrm{H} & 2.383767 & 1.778969 & 0.931687 \\ \mathrm{H} & 2.384207 & 1.778818 & -0.931620 \\ \mathrm{H} & 1.825789 & -1.987564 & 0.000270 \\ \mathrm{H} & -0.433827 & -2.696948 & 0.000052 \\ \mathrm{H} & -2.408439 & -1.425458 & 0.000049 \\ \mathrm{H} & -1.895793 & 2.815241 & -0.000084\end{array}$

$S(I I) C C$

$E(R B L Y P)=309.38683$

$E(\operatorname{RBD}(T)))=308.63818$

$\mathrm{ZPE}=77.64$

$\begin{array}{lrrr}\mathrm{C} & 0.684214 & 0.829387 & -0.000320 \\ \mathrm{C} & -0.684200 & 0.829397 & -0.000032 \\ \mathrm{C} & -1.557852 & -0.342055 & 0.000295 \\ \mathrm{C} & -2.886581 & -0.281265 & 0.000606 \\ \mathrm{C} & -4.198556 & -0.219101 & -0.000124 \\ \mathrm{C} & 1.557848 & -0.342077 & -0.000769 \\ \mathrm{C} & 2.886579 & -0.281313 & -0.000557 \\ \mathrm{C} & 4.198555 & -0.219167 & 0.000691 \\ \mathrm{H} & 1.092435 & -1.336490 & -0.001217 \\ \mathrm{H} & -1.092454 & -1.336474 & 0.001183 \\ \mathrm{H} & -4.778206 & -0.193041 & -0.931782 \\ \mathrm{H} & -4.779165 & -0.190900 & 0.930886 \\ \mathrm{H} & 4.777762 & -0.191473 & 0.932578 \\ \mathrm{H} & 4.779571 & -0.191920 & -0.930091 \\ \mathrm{H} & -1.193160 & 1.798737 & -0.000050 \\ \mathrm{H} & 1.193186 & 1.798721 & -0.000249\end{array}$ 
TS (II) aa

$E($ RBLYP $)=233.22494$

$E(\operatorname{RBD}(T)))=232.66980$

$\mathrm{ZPE}=72.70$

$\begin{array}{lrrr}\text { C } & -0.104349 & 1.251425 & 0.709003 \\ \text { C } & -0.104349 & 1.251425 & -0.709003 \\ \mathrm{C} & 0.185503 & 0.122497 & -1.495411 \\ \mathrm{C} & -0.104349 & -1.213850 & -1.148887 \\ \mathrm{C} & -0.104349 & -1.213850 & 1.148887 \\ \mathrm{C} & 0.185503 & 0.122497 & 1.495411 \\ \mathrm{H} & -0.013525 & 2.236113 & 1.183897 \\ \mathrm{H} & -0.013525 & 2.236113 & -1.183897 \\ \mathrm{H} & 0.832575 & 0.300886 & -2.367170 \\ \mathrm{H} & 0.456650 & -2.011643 & -1.653601 \\ \mathrm{H} & -1.136532 & -1.485784 & -0.949628 \\ \mathrm{H} & 0.456650 & -2.011643 & 1.653601 \\ \mathrm{H} & -1.136532 & -1.485784 & 0.949628 \\ \mathrm{H} & 0.832575 & 0.300886 & 2.367170\end{array}$

TS (II) ab

$E($ RBLYP $)=231.97552$

$E(\operatorname{RBD}(T)))=231.41744$

$\mathrm{ZPE}=57.79$

$\begin{array}{lrrr}\text { C } & -0.155680 & -1.374519 & -0.075759 \\ \text { C } & -1.390175 & -0.692457 & 0.051969 \\ \text { C } & -0.984695 & 1.311204 & -0.110017 \\ \text { C } & 0.287613 & 1.435717 & -0.047715 \\ \text { C } & 1.376922 & 0.562934 & 0.009378 \\ \mathrm{C} & 1.127018 & -0.818168 & 0.161050 \\ \text { H } & 2.390938 & 0.878450 & -0.259810 \\ \text { H } & 1.991467 & -1.491638 & 0.126338 \\ \text { H } & -0.154104 & -2.342447 & -0.594323 \\ \text { H } & -1.874768 & 1.827424 & 0.232911 \\ \text { H } & -2.253974 & -1.124037 & -0.473024 \\ \text { H } & -1.665578 & -0.296011 & 1.034468\end{array}$

TS (II) ac

$E($ RBLYP $)=271.29929$

$E(\operatorname{RBD}(T)))=270.64806$

$\mathrm{ZPE}=75.08$

$\begin{array}{lrrr}\text { C } & 2.536254 & 0.203776 & -0.046133 \\ \text { C } & 1.283362 & -0.245497 & -0.128817 \\ \text { C } & -0.154409 & 1.530796 & 0.260120 \\ \text { C } & -1.392239 & 1.108598 & -0.203921 \\ \text { C } & -1.862030 & -0.210487 & 0.035586 \\ \text { C } & -0.999967 & -1.301062 & 0.163155 \\ \mathrm{C} & 0.412203 & -1.298660 & -0.081714 \\ \text { H } & 3.269173 & -0.257697 & 0.629851 \\ \text { H } & 2.877340 & 1.091372 & -0.588491 \\ \text { H } & -1.978700 & 1.754971 & -0.870639 \\ \text { H } & 0.808953 & -2.248708 & -0.473005 \\ \text { H } & -2.931640 & -0.424458 & -0.072330 \\ \text { H } & -1.459827 & -2.293555 & 0.225694 \\ \text { H } & 0.279709 & 2.481290 & -0.066493 \\ \text { H } & 0.195949 & 1.172003 & 1.225759\end{array}$

TS (II) bb

$\mathrm{E}(\mathrm{RBLYP})=230.74785$

$\mathrm{E}(\mathrm{RBD}(\mathrm{T})))=230.18604$

$\mathrm{ZPE}=42.63$

$\begin{array}{lrrr}\text { C } & 1.229947 & 0.706191 & -0.000023 \\ \text { C } & 1.229723 & -0.706538 & -0.000062 \\ \text { H } & 2.193504 & 1.220608 & -0.000116 \\ \text { H } & 2.193120 & -1.221253 & 0.000147 \\ \text { C } & 0.002771 & 1.387607 & 0.000118 \\ \text { C } & 0.002333 & -1.387589 & 0.000077 \\ \text { C } & -1.220648 & 1.038893 & -0.000022 \\ \text { C } & -1.220998 & -1.038554 & -0.000137 \\ \text { H } & -2.262487 & 1.321558 & -0.000206 \\ \text { H } & -2.262905 & -1.320966 & 0.000472\end{array}$

Ts (II) bc

$\mathrm{E}(\mathrm{RBLYP})=270.05361$

$\mathrm{E}(\operatorname{RBD}(\mathrm{T})))=269.39718$

$\mathrm{ZPE}=60.08$

$\begin{array}{llll}\text { C } & -2.434200 & -0.094344 & 0.053862\end{array}$

C $\quad-1.117813-0.317842-0.096843$

C $\quad-0.125031-1.262263-0.065781$

$\begin{array}{llll}\text { C } & 1.290560 & -1.076538 & 0.082274\end{array}$

$\begin{array}{llll}\text { C } & 1.909991 & 0.164311 & 0.034907\end{array}$

$\begin{array}{llll}\text { C } & 1.058292 & 1.281616 & 0.016822\end{array}$

$\begin{array}{llll}\text { C } & -0.172276 & 1.572680 & -0.046814\end{array}$

$\mathrm{H} \quad-2.975960 \quad-0.467390 \quad 0.932463$

$\mathrm{H} \quad-2.993927 \quad 0.574528 \quad-0.607948$

$\mathrm{H} \quad-0.445400-2.279272 \quad-0.325735$

$\mathrm{H} \quad \begin{array}{llll}\mathrm{H} & 1.911541 & -1.978294 & 0.067709\end{array}$

$\begin{array}{lllll}\mathrm{H} & 2.992609 & 0.250597 & -0.092580\end{array}$

$\begin{array}{llll}\mathrm{H} & -0.946002 & 2.294112 & 0.155538\end{array}$

TS (II) CC

$E(R B L Y P)=309.35882$

$E(\operatorname{RBD}(T)))=308.61011$

$\mathrm{ZPE}=77.32$

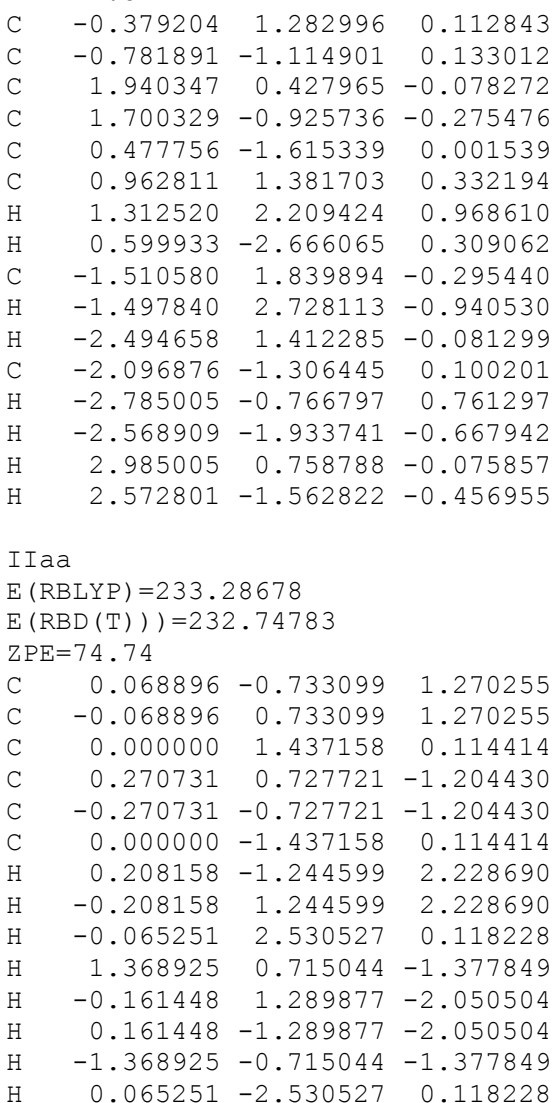


I I ab

$\mathrm{E}(\mathrm{UBLYP})=232.00547$

$E(\operatorname{UBD}(T)))=230,22929$

$\mathrm{ZPE}=42.70$

$<\mathrm{S} 2, \mathrm{UBLYP}>=0.000$

$<\mathrm{S} 2, \mathrm{UBD}(\mathrm{T})>=0.000$

$\begin{array}{lrrr}\text { C } & 0.065142 & 1.313451 & -0.054348 \\ \text { C } & -1.265502 & 0.593350 & 0.234420 \\ \text { C } & -1.157507 & -0.879662 & -0.170095 \\ \text { C } & 0.070237 & -1.397866 & -0.020268 \\ \text { C } & 1.254098 & -0.780023 & 0.160637 \\ \text { C } & 1.247862 & 0.657895 & -0.212741 \\ \text { H } & 2.085261 & -1.177257 & 0.755663 \\ \text { H } & 2.177962 & 1.203330 & -0.406008 \\ \text { H } & 0.035160 & 2.408756 & -0.075995 \\ \text { H } & -1.945990 & -1.327081 & -0.785651 \\ \text { H } & -1.575728 & 0.815041 & 1.279667 \\ \text { H } & -2.062648 & 1.034342 & -0.393304\end{array}$

I I ac

$E($ RBLYP $)=271.36886$

$E(\operatorname{RBD}(T)))=270.73293$

$\mathrm{ZPE}=77.43$

$\begin{array}{lrrr}\text { C } & -1.258544 & -1.151310 & -0.054877 \\ \mathrm{C} & 0.243158 & -1.298451 & 0.112687 \\ \mathrm{C} & 1.041071 & 0.015794 & 0.015790 \\ \mathrm{C} & 0.283085 & 1.267829 & 0.063548 \\ \mathrm{C} & -1.080644 & 1.294252 & 0.024639 \\ \mathrm{C} & -1.863928 & 0.059908 & -0.072589 \\ \mathrm{C} & 2.394915 & 0.017877 & -0.097456 \\ \mathrm{H} & -1.611007 & 2.251448 & 0.038740 \\ \mathrm{H} & -2.952033 & 0.137045 & -0.164052 \\ \mathrm{H} & -1.845018 & -2.073754 & -0.122453 \\ \mathrm{H} & 0.854615 & 2.201720 & 0.094216 \\ \mathrm{H} & 2.961132 & 0.953560 & -0.124244 \\ \mathrm{H} & 2.974594 & -0.907768 & -0.162413 \\ \mathrm{H} & 0.430009 & -1.762791 & 1.105037 \\ \mathrm{H} & 0.633030 & -2.034859 & -0.615283\end{array}$

I Ibb

$E($ UBLYP $)=230.77789$

$\mathrm{E}(\mathrm{UBD}(\mathrm{T})))=230.21939$

$\mathrm{ZPE}=44.78$

$\langle\mathrm{S} 2, \mathrm{UBLYP}\rangle=0.804$

$<\mathrm{S} 2, \mathrm{UBD}(\mathrm{T})>=1.810$

$\begin{array}{llll}\text { C } & 0.000000 & 0.000000 & 1.359735\end{array}$

$\begin{array}{llll}\text { C } & 0.000000 & 1.221516 & 0.722727\end{array}$

C $\quad 0.000000 \quad 1.221516-0.722727$

C $\quad 0.000000 \quad 0.000000-1.359735$

C $\quad 0.000000-1.221516-0.722727$

$\begin{array}{llll}\text { C } & 0.000000 & -1.221516 & 0.722727\end{array}$

$\begin{array}{llll}\mathrm{H} & 0.000000 & 2.183388 & 1.243424\end{array}$

$\mathrm{H} \quad 0.000000 \quad 2.183388-1.243424$

$\mathrm{H} \quad 0.000000 \quad-2.183388-1.243424$

$\mathrm{H} \quad 0.000000 \quad-2.183388 \quad 1.243424$
IIbc

$\mathrm{E}(\mathrm{UBLYP})=270.10153$

$E(\operatorname{UBD}(T)))=269.45450$

$\mathrm{ZPE}=61.77$

$<\mathrm{S} 2, \mathrm{UBLYP}>=1.027$

$<\mathrm{S} 2, \mathrm{UBD}(\mathrm{T})>=1.733$

$\begin{array}{llll}\text { C } & 0.076094 & 2.382835 & 0.000000\end{array}$

C $\quad 0.000000 \quad 0.975575 \quad 0.000000$

C $\quad 1.182886 \quad 0.151416 \quad 0.000000$

C $\quad 1.110794-1.241513 \quad 0.000000$

C $\quad-0.148594-1.905105 \quad 0.000000$

C $\quad-1.273320-1.084043 \quad 0.000000$

$\begin{array}{llll}\text { C } & -1.279695 & 0.289572 & 0.000000\end{array}$

H $\quad 1.039884 \quad 2.897285 \quad 0.000000$

$\mathrm{H} \quad-0.825227 \quad 2.999720 \quad 0.000000$

H $\quad 2.159183 \quad 0.645581 \quad 0.000000$

H $\quad 2.030857-1.835853 \quad 0.000000$

$\mathrm{H} \quad-0.210393-2.996854 \quad 0.000000$

$\mathrm{H} \quad-2.203291 \quad 0.877704 \quad 0.000000$

I ICC

$E(R B L Y P)=309.45496$

$E(\operatorname{RBD}(T)))=308.72339$

$\mathrm{ZPE}=80.65$

$\begin{array}{lrrr}\text { C } & -0.680703 & 1.419043 & 0.092504\end{array}$

$0.623514 \quad 0.753739-0.000061$

$0.623514-0.7537390 .000061$

$-0.680702-1.419043-0.092504$

$-1.855817-0.725194-0.057980$

$\begin{array}{llll}-1.855817 & 0.725194 & 0.057980\end{array}$

$1.754157 \quad 1.511715-0.116492$

$1.754157-1.511714 \quad 0.116492$

$-2.811887-1.255077-0.104561$

$\begin{array}{lll}-2.811887 & 1.255077 & 0.104561\end{array}$

$\begin{array}{lll}-0.681170 & 2.511897 & 0.155698\end{array}$

$-0.681170-2.511897-0.155698$

$2.747407 \quad 1.078929-0.252558$

$\begin{array}{lll}1.698740 & 2.603182-0.089440\end{array}$

$1.698740-2.603182 \quad 0.089440$

$2.747408-1.078929 \quad 0.252558$ 